\title{
Efectividad de intervenciones basadas en Mindfulness para mejorar el control de la Diabetes Mellitus tipo 2: Una revisión sistemática e integración metanalitica preliminar
}

\section{Effectiveness of Mindfulness-based interventions to improve control of type 2 diabetes mellitus: A systematic review and preliminary meta-analytic integration}

\author{
Alexis González-Burboa, MSc, $\mathrm{PhD}(\mathrm{c})$ \\ Departamento de Salud Pública, Facultad de Medicina, Universidad de Concepción, Chile. \\ Programa de Salud y Medicina Familiar, Facultad de Medicina, Universidad de Concepción, Chile. \\ Soledad Luarte-Martínez, MSc \\ Departamento de Kinesiología, Facultad de Medicina, Universidad de Concepción, Chile. \\ Patricia Villaseca Silva, MD \\ Departamento de Salud Pública, Facultad de Medicina, Universidad de Concepción, Chile. \\ Programa de Salud y Medicina Familiar, Facultad de Medicina, Universidad de Concepción, Chile. \\ Camilo Manríquez Vidal, Lic \\ Departamento de Obstetricia y Ginecología, Facultad de Medicina, Universidad de Concepción, Chile.
}

Hans Müller Ortiz, MD

Servicio de Nefrología, Hospital Las Higueras de Talcahuano, Talcahuano, Chile.

Departamento de Medicina Interna, Facultad de Medicina, Universidad de Concepción, Chile.

Cristian Pedreros Rosales, MD

Unidad Paciente Crítico Adulto, Hospital Las Higueras de Talcahuano, Talcahuano, Chile. Departamento de Medicina Interna, Facultad de Medicina, Universidad de Concepción, Chile.

Ángel Alberquilla Menéndez-Asenjo, MD, PhD

Departamento de Medicina Preventiva y Salud Pública, Facultad de Medicina, Universidad Autónoma de Madrid, España.

Ángel Otero Puime, MD, PhD

Departamento de Medicina Preventiva y Salud Pública, Facultad de Medicina, Universidad Autónoma de Madrid, España.

Darío Páez Rovira, $\mathrm{PhD}$

Departamento de Psicología Social y Metodología, Facultad de Psicología, Universidad del País Vasco, España.

Aldo Vera-Calzaretta, $\mathrm{PhD}$

Departamento de Salud Pública, Facultad de Medicina, Universidad de Concepción, Chile. Programa de Salud y Medicina Familiar, Facultad de Medicina, Universidad de Concepción, Chile. 
Financiamiento: Este estudio se enmarca dentro del proyecto VRID 217.089.006-1.0IN financiado por la Vicerrectoría de Investigación y Desarrollo de la Universidad de Concepción, Chile. No existen conflictos de interés con la fuente de financiamiento de esta investigación.

\title{
Resumen
}

El propósito de este trabajo fue estudiar la efectividad de intervenciones basadas en Mindfulness sobre el nivel de hemoglobina glicada - HbA1c - en pacientes con diabetes mellitus tipo 2 - DM2 - Se realizó una revisión sistemática e integración metanalítica preliminar. La búsqueda de los estudios se realizó en las siguientes bases: PubMed, Bireme, Web of Science, SciELO, Embase, EBSCOhost, SCOPUS, Psychology Database. Se identificaron 10 artículos: cuatro fueron llevados a cabo en Estados Unidos, dos en Irán, uno en Alemania, uno en Australia, uno en Tailandia y uno en Inglaterra. Se observó una reducción en los niveles de la HbA1c utilizando Mindfulness en comparación a los grupos controles $(\mathrm{p}<0,02)$. Además, se observó un efecto diferenciado al analizar según número de participantes, sexo y tiempo de seguimiento. Se concluyó que el uso de intervenciones basadas en Mindfulness tendría un efecto indirecto sobre la reducción de la hemoglobina glicada.

Palabras clave: Diabetes Mellitus Tipo 2, Hemoglobina A Glicada, Mindfulness (DeCS, BVS)

\begin{abstract}
The purpose of this paper was to study the effectiveness of Mindfulness-based interventions on the level of glycated hemoglobin - HbA1c — in patients with type 2 diabetes mellitus - DM2 - . A systematic review and preliminary meta-analytic integration was performed. The search of the studies was carried out in the following bases: PubMed, Bireme, Web of Science, SciELO, Embase, EBSCOhost, SCOPUS, Psychology Database. Ten articles were identified: four were published in the United States, two in Iran, one in Germany, one in Australia, one in Thailand and one in England. A reduction in HbA1c levels was observed using Mindfulness compared to control groups $(p<0,02)$. In addition, according to the number of participants, sex and time of follow-up a differentiated effect was found. It was concluded that the use of interventions based on Mindfulness would have an indirect effect on the reduction of glycated hemoglobin (HbA1c).
\end{abstract}

Keywords: Type 2 Diabetes

Mellitus; Glycated Hemoglobin

A; Mindfulness (MeSH, NHL)

\section{Introducción}

Según los datos provistos por la Federación Internacional de la Diabetes, se puede afirmar que esta enfermedad se ha constituido en una de las principales pandemias del siglo XXI. Actualmente, se estima que su prevalencia para el grupo comprendido entre los 20 y 79 años es de $8,8 \%$ y que para el año 2040 la prevalencia de la población mundial será de 10,4\%. Dentro de las distintas formas en que se expresa la enfermedad, la más frecuente es la diabetes mellitus tipo 2 -DM2-, cuyas prevalencias en los países desarrollados oscila entre un $87 \%$ y un $91 \%$ (International Diabetes Federation, 2015, 2017).

Dada la complejidad de dicho tipo de condición crónica, para responder a esta problemática, los modelos de cuidado han ido incorporando distintos tipos de estrategias de abordaje terapéutico, complementarias a aquellos paradigmas focalizados en atender aspectos netamente biomédicos
(Comunidad de Madrid, 2013; Organización Panamericana de la Salud, 2013). Al respecto, se ha dado especial atención al desarrollo de estrategias abocadas al fortalecimiento de la autogestión de las personas, el apoyo a la toma de decisiones y el resguardo de aspectos vinculados a las prestaciones otorgadas, entre otros (Bodenheimer, Wagner, \& Grumbach, 2002; Piatt et al., 2006; Singh \& Ham, 2008; Wagner, 1998; Wagner et al., 2001; Wagner, Austin, \& Von Korff, 1996).

En este contexto, se ha estudiado el efecto que distintas intervenciones psicológicas podrían tener sobre la hemoglobina glicada - $\mathrm{HbAlc}$-, encontrándose disminuciones significativas en sus niveles (Alam, Sturt, Lall, \& Winkley, 2009; Ismail, Winkley, \& Rabe-Hesketh, 2004). Lo anterior, releva la necesidad de generar evidencia acerca de intervenciones de bajo costo que puedan ser ampliamente utilizadas en el escenario de cuidados de personas con DM2. Tal es el caso de aquellas que incorporan el Mindfulness como parte de su diseño. Según la evidencia, estas contribuirían a mejorar la auto-regulación emocional, la empatía, la compasión, las habilidades de consejería y a disminuir el estrés y la ansiedad, tanto en quienes reciben la intervención, así como, en quienes la aplican (Davis \& Hayes, 2011). 
El Mindfulness se ha conceptualizado como "un estado psicológico de conciencia, una práctica que promueve esta conciencia, un modo de procesar la información, un rasgo del carácter" (Davis \& Hayes, 2011). La práctica de éste permite dirigir la conciencia al momento presente, dándose cuenta y aceptando lo que ocurre sin juzgarlo (Vandenberg et al., 2018). Su aplicación ha sido útil para el manejo de distintos problemas relacionados con la salud. Por ejemplo, en una revisión sistemática que evaluó el efecto del programa Mindfulness-Based Stress Reduction - MBSR - sobre variables psicológicas en personas que se encontraban en terapia para afrontar el cáncer, se halló que éste contribuyó a mejorar su calidad de vida, bienestar emocional y a reducir el estrés. En este caso, la intervención grupal duró 8 semanas e incorporó una combinación de meditación Mindfulness y ejercicios de yoga, y adicionalmente, ofreció una sesión de meditación en silencio (Smith, Richardson, Hoffman, \& Pilkington, 2005).

En otro estudio experimental aleatorizado, luego de haber implementado un programa de 4 meses que integró componentes del MBSR y del programa de entrenamiento Mindfulness-Based Eating Awareness Training MB-EAT- - se reportó una mejora en la acción enzimática, en los niveles de distress y en el comportamiento alimentario de personas con malnutrición por exceso. En el referido programa se instruyó a los participantes para que realizaran una meditación de forma sentada, escaneo corporal, ejercicios de yoga y prácticas de alimentación consciente, las cuales, consistieron en prestar atención a las sensaciones de hambre, de saciedad y de satisfacción con el gusto, entre otras (Daubenmier et al., 2012).

Finalmente, en un metanálisis de ensayos clínicos randomizados, cuyo objetivo fue evaluar el efecto de la MBSR sobre la salud mental de adultos con trastornos crónicos de tipo somático, se reportaron efectos significativos sobre la depresión, la ansiedad y el distress (Bohlmeijer, Prenger, Taal, \& Cuijpers, 2010). No obstante lo anterior, al momento de esta revisión no se encontraron trabajos que sintetizaran la evidencia de intervenciones que incorporaran el Mindfulness y que evaluaran su efecto en los niveles de HbAlc como coadyuvante en el manejo de personas con DM2.

En consecuencia, el propósito de este estudio fue llevar a cabo una revisión sistemática, y posterior integración metanalítica preliminar, de estudios que reportaron el efecto de intervenciones que incorporaron el Mindfulness como parte de su diseño sobre los niveles de $\mathrm{HbA} 1 \mathrm{c}$ en personas con DM2.

\section{Método}

\section{Diseño}

Se llevó a cabo una revisión sistemática, y posterior integración metanalítica preliminar, de estudios que reportaron el efecto de intervenciones que incorporaron el Mindfulness como parte de su diseño sobre los niveles de $\mathrm{HbAlc}$ en personas con DM2. Para la realización de este trabajo, se siguieron los criterios establecidos por la guía PRISMA (Preferred Reporting Items for Systematic Reviews and Meta-Analyses) (Liberati et al., 2009).

\section{Proceso de búsqueda de la literatura}

La búsqueda de los estudios fue realizada, dadas las posibilidades de acceso, en las siguientes bases de datos electrónicas: PubMed, Bireme, Web of Science, SciELO, Embase, EBSCOhost, SCOPUS, Psychology Database, desde sus inicios hasta el 31 de junio de 2018. Se utilizaron los operadores boléanos AND y OR. Para las bases en inglés, se usó los términos MeSH (Medical Subjects Headings) "Mindfulness", "Diabetes Melltius, Type 2" y "Glycated Hemoglobin A". Además, se utilizó los términos libres "mindfulness", "glycated hemoglobin", "diabetes mellitus type 2", "non-insulin-dependent diabetes" y "glycosylated hemoglobin". Específicamente, en la base Embase se aplicaron los límites de búsqueda "full text", "human", "english language", "article" y "adult $<18$ to 64 years $>$ or aged $<65+$ years $>$ ". Por otra parte, si las bases de datos estaban en idioma español, se usó los términos DeCS (Descriptores en Ciencias de la Salud) "Diabetes Mellitus Tipo 2", "Hemoglobina A Glicada" y "Mindfulness". A continuación se presenta el flowchart del proceso de búsqueda bibliográfica (Fig. 1).

\section{Criterios de elegibilidad de los estudios}

Se incluyeron estudios experimentales, que evaluaron el efecto de intervenciones basadas en Mindfulness sobre los niveles de $\mathrm{HbA} 1 \mathrm{c}$ en pacientes con diabetes tipo 2. Se consideraron como criterios de elegibilidad: (a) pacientes con una edad mayor o igual a 18 años; (b) diabetes mellitus tipo 2; (c) intervenciones que incorporaron el Mindfulness como parte de su diseño; y (d) tener como una variable de resultado el nivel de $\mathrm{HbAlc}$. 
Figura 1. Flow chart PRISMA de los estudios seleccionados

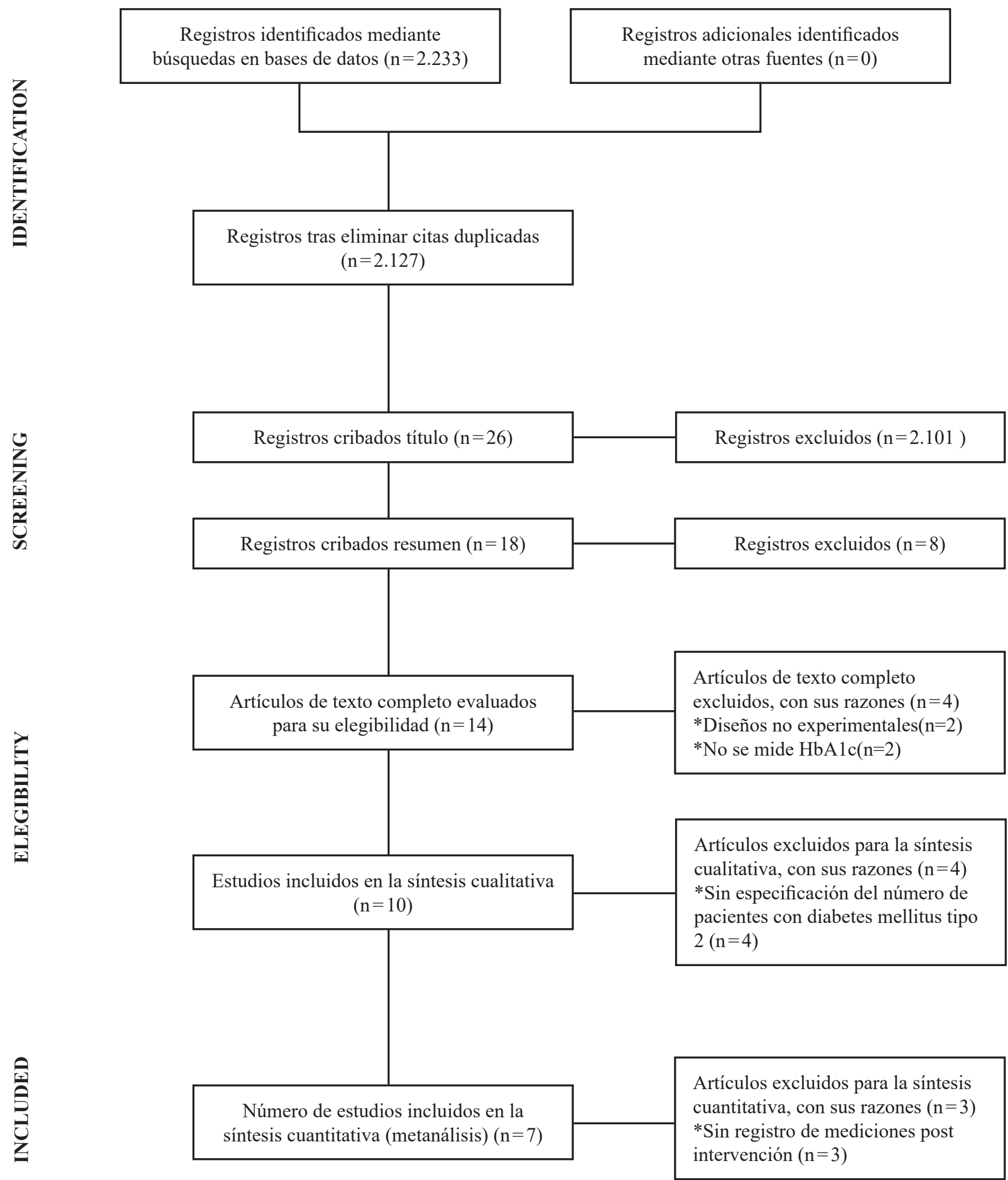




\section{Extracción de los datos.}

Los datos fueron extraídos por dos revisores independientes, registrándose: (a) autor, año, referencia del estudio, país de realización del estudio; (b) $\mathrm{n}^{\circ}$ de participantes en la línea base y al final del seguimiento; (c) media y desviación estándar de la edad en el grupo de intervención (expresada en años), proporción de hombres; (d) tipo y duración de la intervención; (e) régimen (sesiones) en el grupo de intervención, tipo de terapeuta; (f) media y desviación estándar del \% de $\mathrm{HbAlc}$ en el grupo de intervención en la línea base; (g) media y desviación estándar del \% de HbAlc en el grupo de intervención al final seguimiento; (h) seguimiento el término de la intervención, expresado en meses; (i) media y desviación estándar de la edad en el grupo de control (expresada en años), proporción de hombres; (j) tipo y duración de la terapia en el grupo control, (k) régimen (sesiones) en el grupo control y tipo de terapeuta; (l) media y desviación estándar del \% de HbAlc en el grupo control en la línea base; (m) media y desviación estándar de la HbA1c en el grupo control al final seguimiento; (n) resultados; y (o) calidad metodológica del estudio.

Sumado a lo anterior, se extrajeron los siguientes datos de mediciones en variables psicológicas: (a) media $\pm \mathrm{DE}$ en el grupo de intervención en la línea base; (b) media $\pm \mathrm{DE}$ en el grupo de intervención al final del seguimiento; (c) media $\pm \mathrm{DE}$ en el grupo control en la línea base; (d) media $\pm \mathrm{DE}$ en el grupo control final del seguimiento; y (e) resultados.

Para aquellos casos en los cuales hubo desacuerdo, los artículos se revisaron en forma conjunta hasta lograr llegar a consenso.

\section{Evaluación de la calidad metodológica y sesgo de publicación}

La evaluación de la calidad metodológica de los trabajos incluidos en la síntesis cualitativa de este estudio, fue llevada a cabo por dos evaluadores independientes. Para este efecto, se utilizó la escala de Jadad (Koloverou, Tentolouris, Bakoula, Darviri, \& Chrousos, 2014) que evalúa los siguientes criterios en los ensayos clínicos: aleatorización, enmascaramiento y perdidas de seguimiento. Esta escala consta de 3 ítems cuya respuesta correcta equivale a 1 punto, e incorrecta a 0 puntos. Además, consta de dos preguntas adicionales que indagan la idoneidad de los procesos de aleatorización y enmascaramiento. La puntuación total de esta escala va de 0 a 5 puntos, estableciéndose que la obtención de un puntaje más alto refleja una mejor calidad metodológica del estudio. En este respecto, se considera que un puntaje menor a 3 indica una baja calidad metodológica.

El nivel de acuerdo entre los evaluadores fue establecido mediante la prueba de concordancia de Kappa. Para resol- ver aquellos casos en los cuales no hubo acuerdo, se incluyó la opinión de un tercer evaluador independiente.

\section{Análisis estadísticos}

Para la integración metanalítica, de los estudios incluidos se extrajeron los siguientes datos: (a) media, (b) desviación estándar y (c) tamaño muestral.

La unidad de medida del nivel de HbA1c de los estudios se expresó en porcentaje (\%), no obstante, un trabajo (Gainey, Himathongkam, Tanaka \& Suksom, 2016) reportó la $\mathrm{HbAlc}$ en $\mathrm{mmol} / \mathrm{mol}$, por lo cual, el valor se transformó a $\%$ utilizando la siguiente fórmula (Blot et al., 1988):

Por otra parte, en dos estudios (Murray et al., 2017; Saslow et al., 2017) no se reportaron las desviaciones estándar de las medidas post intervención, por tanto, se asumieron los valores de la medición de línea base.

Para la integración metanalítica se utilizaron dos estrategias. La primera, consistió en la estimación de las diferencias de medias usando un modelo de efectos fijos y un modelo de efectos aleatorios. La segunda, con el propósito de estudiar la consistencia de los resultados, consideró la estimación de las diferencias de medias estandarizadas, también aplicando los mismos modelos de análisis.

Para evaluar la heterogeneidad de los estudios se utilizó el test de $\mathrm{Chi}^{2}$ y el estadístico $\mathrm{I}^{2}$. En relación a este último, los valores fueron interpretados en función de las siguientes categorías: adecuada ( $0 \%$ a $40 \%)$, moderada (30\% a $60 \%$ ), sustancial (50 a $90 \%)$, o considerable ( $75 \%$ a $100 \%$ ) (Wang, Coleman, Kanter, Ummer \& Siminerio, 2018).

Por otra parte, con el fin de explorar las posibles fuentes de heterogeneidad, se realizó un análisis preliminar de subgrupos según las siguientes variables calculando la diferencia de medias estandarizadas mediante el modelo de efectos aleatorios: número de participantes en el grupo de intervención ( $<30$ casos, 30 ó más casos), proporción de hombres y de mujeres ( $>$ proporción de hombres, $>$ proporción de mujeres), tipo de intervención (individual auto-aplicada, grupal con facilitador), duración de la intervención ( $8 \mathrm{se}-$ manas o menos, $>8$ semanas) y la duración del seguimiento al finalizar la intervención ( $<12$ meses, 12 meses).

Para el análisis de los datos se utilizó el software Review Manager V.5.3. La significancia estadística se estableció con un valor de $\mathrm{p} \leq 0.05$.

\section{Resultados}

\section{Sintesis cualitativa de los estudios}

Se identificaron 10 estudios (Armani Kian et al., 2018; Chacko, Yeh, Davis \& Wee, 2016; Gainey, et al., 2016; Gre- 
gg, Callaghan, Hayes, \& Glenn-Lawson, 2007; Hartmann et al., 2012; Murray, et al., 2017; Pearson, Wills, Woods, \& Warnecke, 2018; Putiri et al., 2012; Saslow, et al., 2017; Shayeghian, Hassanabadi, Aguilar-Vafaie, Amiri \& Besharat, 2016), de los cuales, cuatro fueron llevados a cabo en Estados Unidos, dos en Irán, uno en Alemania, uno en Australia, uno en Tailandia y uno en Inglaterra.

La edad promedio de los participantes estuvo comprendida entre los 51,9 y 64,9 años. La mayoría de los estudios reportó la proporción de hombres y mujeres en función de los casos incluidos en el grupo control y el grupo de intervención. Solo un trabajo no dio cuenta de esta diferencia (Putiri, et al., 2012).

Respecto a las intervenciones que incorporaron Mindfulness, mayoritariamente las sesiones duraron entre 8 a 12 semanas (Armani Kian, et al., 2018; Chacko, et al., 2016; Gainey, et al., 2016; Hartmann, et al., 2012; Murray, et al., 2017; Pearson, et al., 2018; Putiri, et al., 2012). También hubo una intervención que duró 32 semanas (Saslow, et al., 2017) y una que duró 4 horas (Gregg, et al., 2007). La mayoría, fueron intervenciones grupales mediadas por un facilitador (Armani Kian, et al., 2018; Chacko, et al., 2016; Gainey, et al., 2016; Gregg, et al., 2007; Hartmann, et al., 2012; Putiri, et al., 2012; Shayeghian, et al., 2016), no obstante, en tres estudios las intervenciones se llevaron a cabo utilizando recursos virtuales sin un facilitador. El primero, utilizó un CD de auto-instrucción para la respiración consciente guiada (Pearson, et al., 2018), el segundo, un programa basado en la web con recomendaciones para una dieta ketogénica baja y estilos de vida saludable, y el tercero, un programa on-line autodirigido (Murray, et al., 2017).

El tiempo de seguimiento de los participantes una vez finalizada la intervención, fue de: (a) 3 meses en cinco estudios (Armani Kian, et al., 2018; Gainey, et al., 2016; Gregg, et al., 2007; Pearson, et al., 2018; Shayeghian, et al., 2016); (b) 12 meses en dos estudios (Hartmann, et al., 2012; Murray, et al., 2017); y (c) 6 meses en un estudio (Chacko, et al., 2016). En dos trabajos no hubo seguimiento una vez finalizada la intervención (Putiri, et al., 2012; Saslow, et al., 2017).

Al comparar los resultados pre y post de los grupos que incorporaron el Mindfulness, en la mayor parte de los estudios, se reportó una disminución significativa del nivel de HbAlc al finalizar la intervención (Armani Kian, et al., 2018; Chacko, et al., 2016; Murray, et al., 2017; Pearson, et al., 2018; Saslow, et al., 2017; Shayeghian, et al., 2016). En cambio, en los grupos controles la mayoría de los estudios reportaron un aumento en los niveles de HbAlc (Gainey, et al., 2016; Hartmann, et al., 2012; Murray, et al., 2017; Shayeghian, et al., 2016). Por otra parte, dos estudios mostraron disminuciones (Gregg, et al., 2007; Saslow, et al., 2017) y uno no presentó variaciones (Pearson, et al., 2018). Cabe señalar, que tres estudios no presentaron datos de los niveles de $\mathrm{HbAlc}$ post intervención (Armani Kian, et al., 2018; Chacko, et al., 2016; Putiri, et al., 2012).

Al comparar los resultados obtenidos al final del seguimiento entre los grupos de intervención y control, la mayor parte de los estudios mostró una disminución estadísticamente significativa de la $\mathrm{HbA} 1 \mathrm{c}$ a favor de la inter- 
Alexis González-Burboa, Soledad Luarte-Martínez, Patricia Villaseca, Camilo Manríquez, Hans Müller, Cristian Pedreros, Ángel Menéndez-Asenjo, Ángel Otero, Darío Páez y Aldo Vera-Calzaretta

\begin{tabular}{|c|c|c|}
\hline 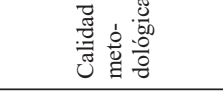 & $\frac{\pi}{4}$ & 舀 \\
\hline 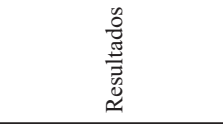 & 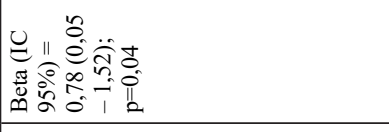 & 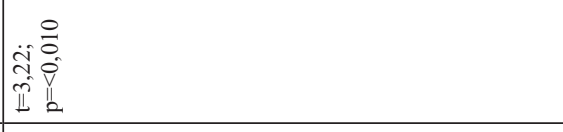 \\
\hline 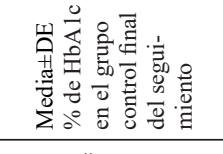 & 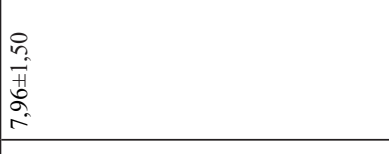 & $\tilde{z}$ \\
\hline 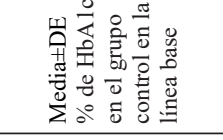 & 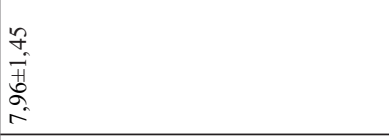 & 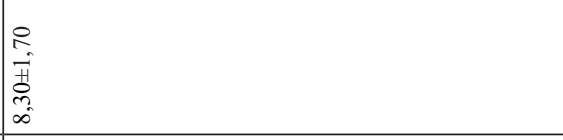 \\
\hline 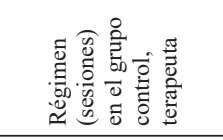 & $\frac{\tilde{z}}{z}$ & $\frac{\tilde{z}}{z}$ \\
\hline 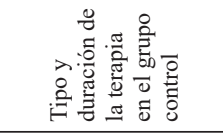 & 苂 & 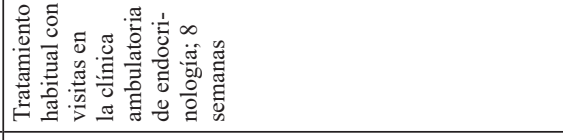 \\
\hline 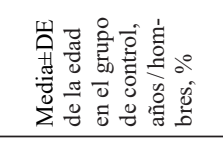 & 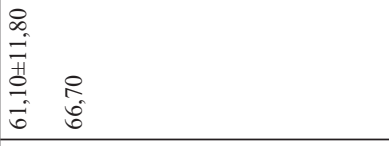 & 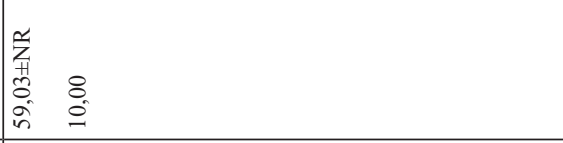 \\
\hline 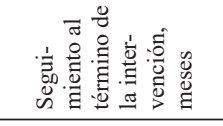 & 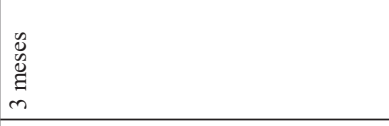 & 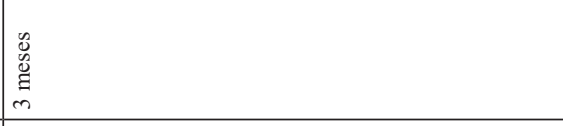 \\
\hline 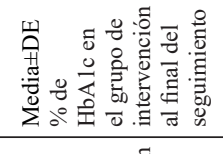 & 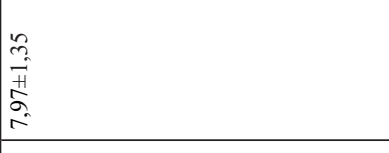 & $\frac{\tilde{z}}{z}$ \\
\hline 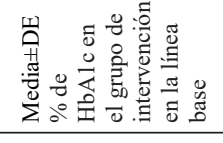 & 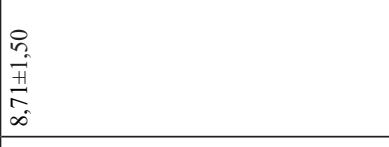 & 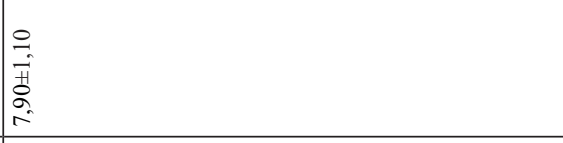 \\
\hline 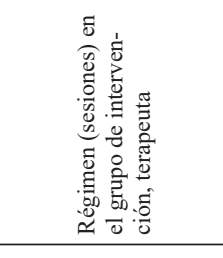 & 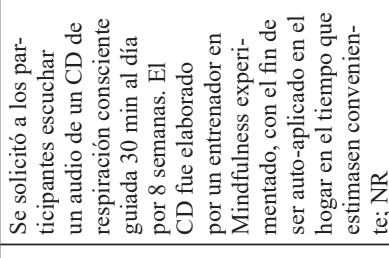 & 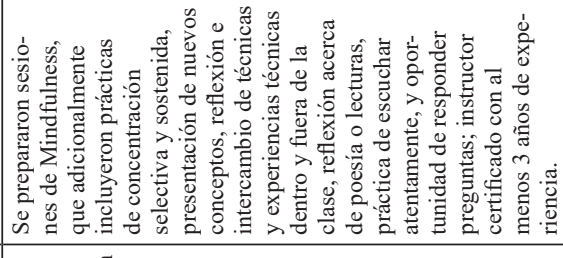 \\
\hline 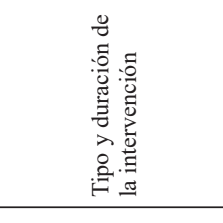 & 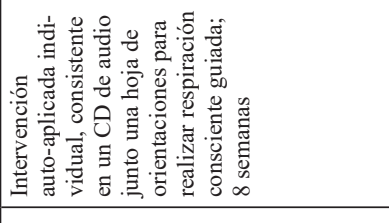 & 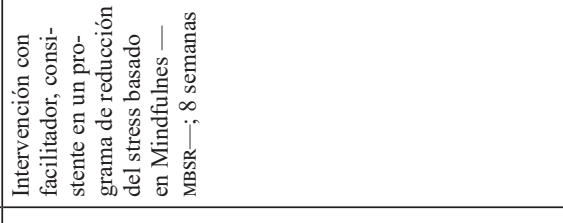 \\
\hline 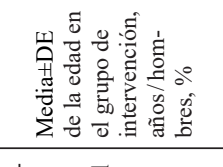 & 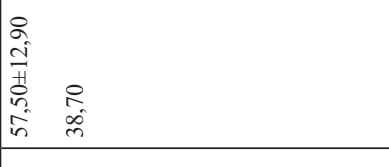 & 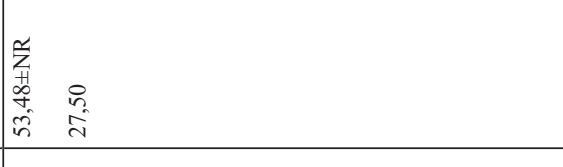 \\
\hline 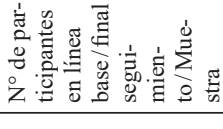 & 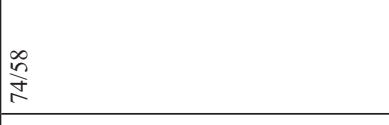 & 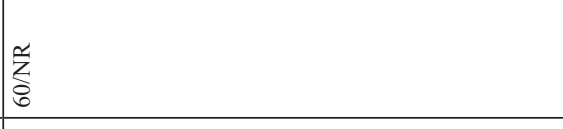 \\
\hline 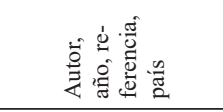 & 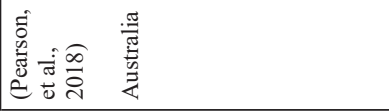 & 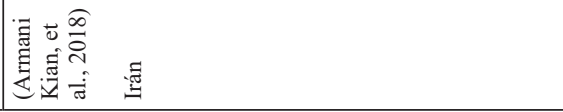 \\
\hline
\end{tabular}




\begin{tabular}{|c|c|c|}
\hline 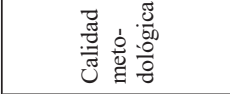 & $\frac{\pi}{2}$ & $\frac{\pi}{4}$ \\
\hline 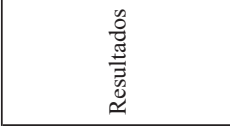 & 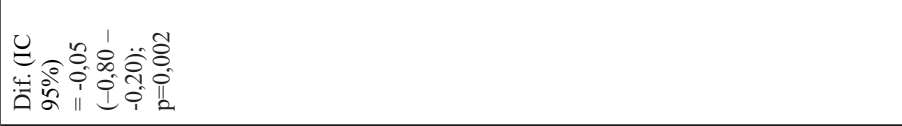 & 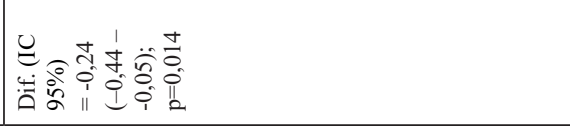 \\
\hline 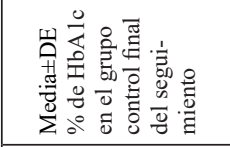 & 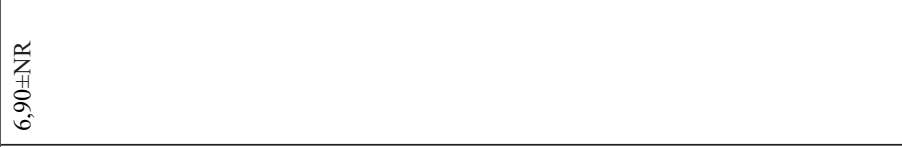 & 旁 \\
\hline 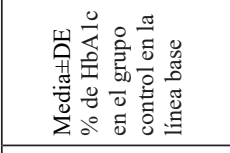 & 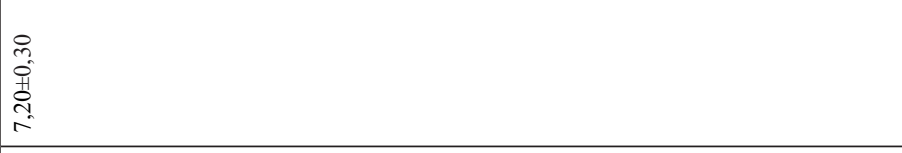 & $\begin{array}{l}0 \\
0 \\
01 \\
0 \\
0 \\
\\
\end{array}$ \\
\hline 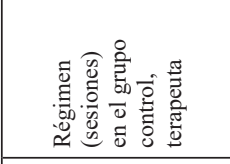 & 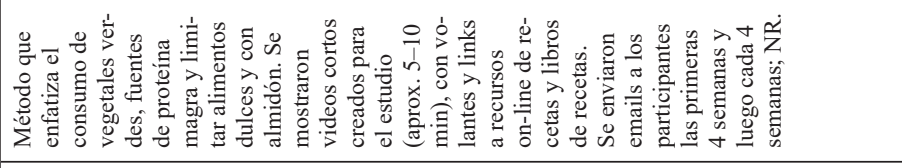 & 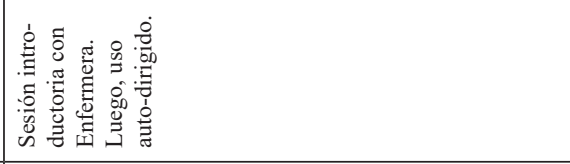 \\
\hline 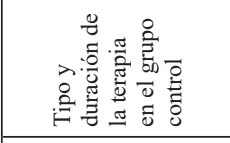 & 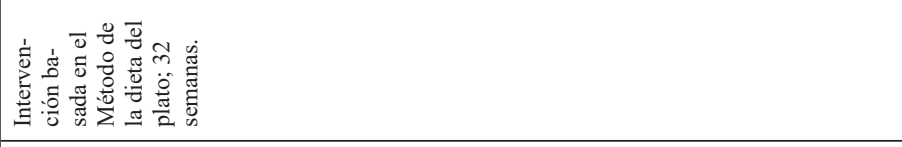 & 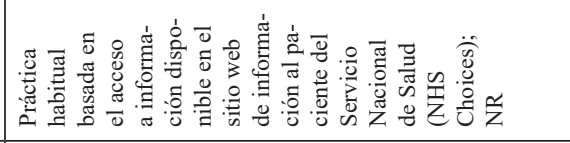 \\
\hline 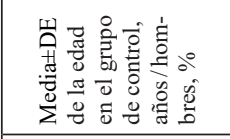 & 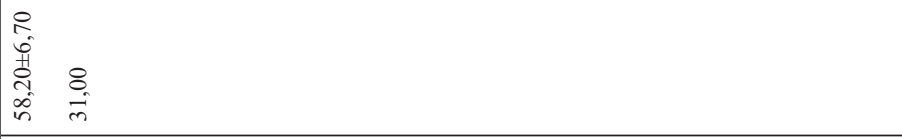 & 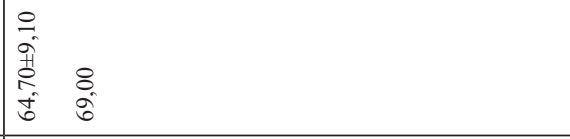 \\
\hline 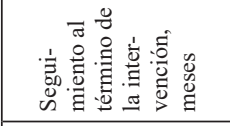 & 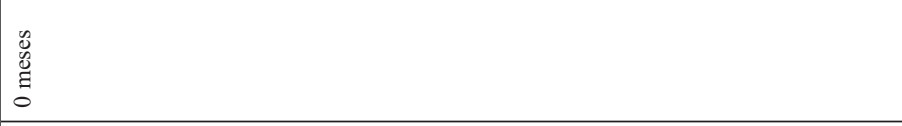 & 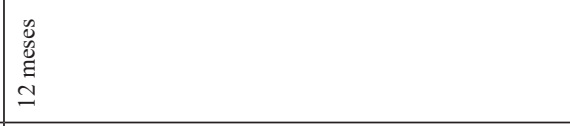 \\
\hline 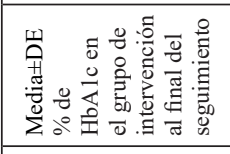 & 总 & 禀 \\
\hline 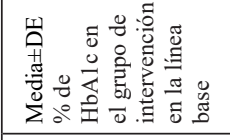 & 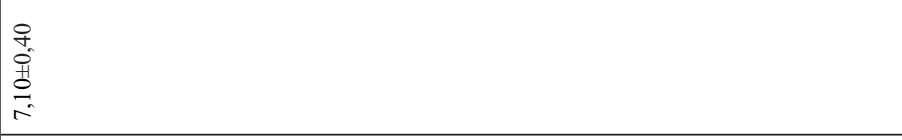 & 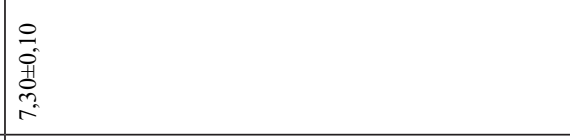 \\
\hline 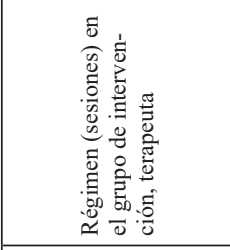 & 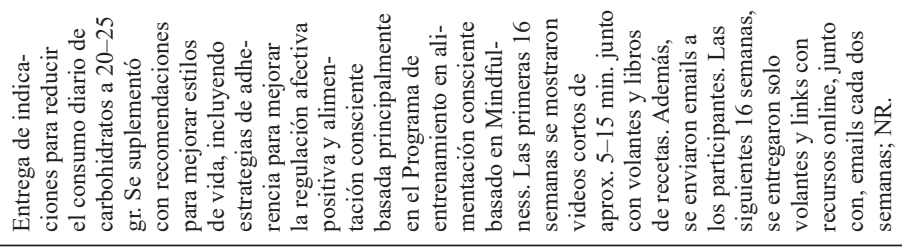 & 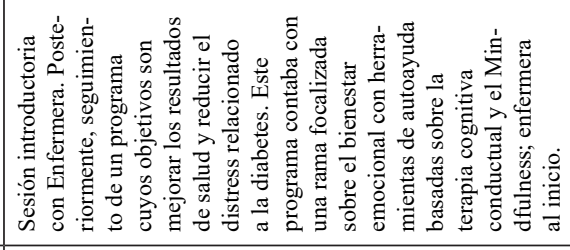 \\
\hline 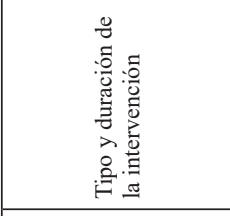 & 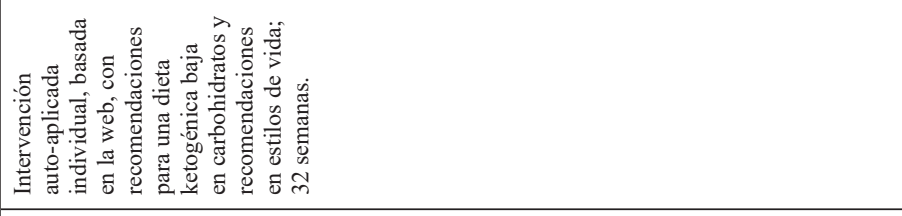 & 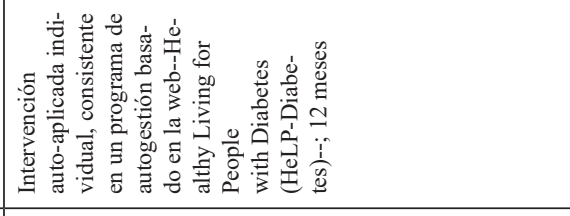 \\
\hline 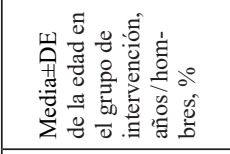 & 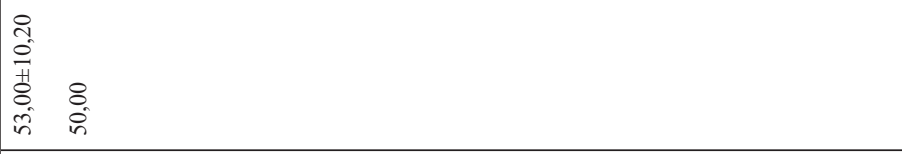 & 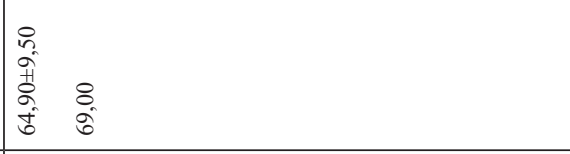 \\
\hline 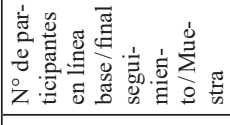 & ने & 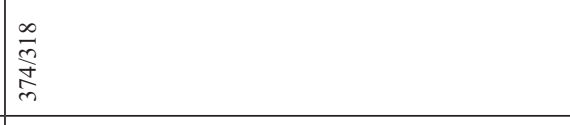 \\
\hline 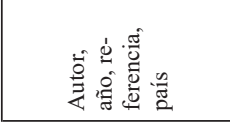 & 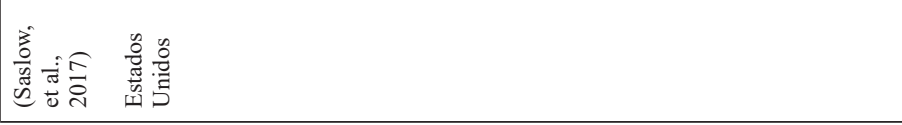 & 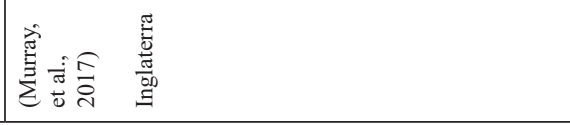 \\
\hline
\end{tabular}


Alexis González-Burboa, Soledad Luarte-Martínez, Patricia Villaseca, Camilo Manríquez, Hans Müller, Cristian Pedreros, Ángel Menéndez-Asenjo, Ángel Otero, Darío Páez y Aldo Vera-Calzaretta

\begin{tabular}{|c|c|}
\hline 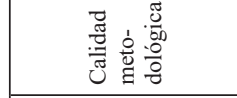 & $\frac{\pi}{4}$ \\
\hline 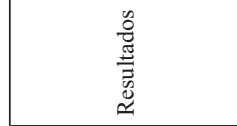 & 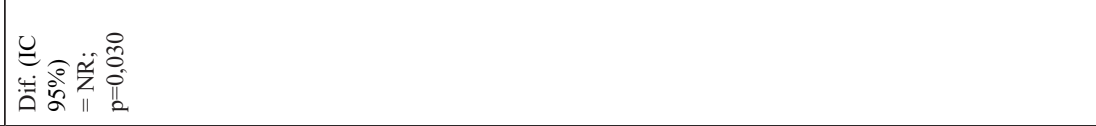 \\
\hline 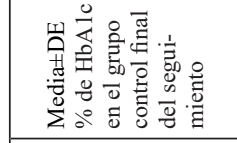 & 苂 \\
\hline 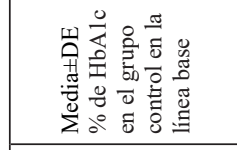 & $\tilde{z}$ \\
\hline 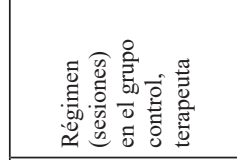 & 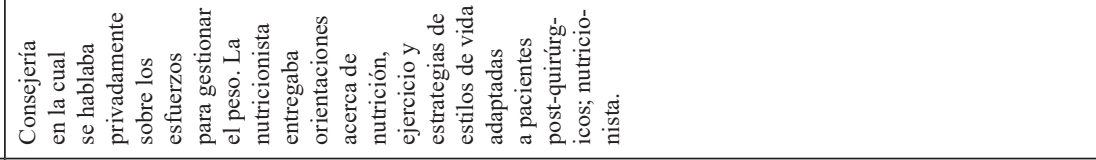 \\
\hline 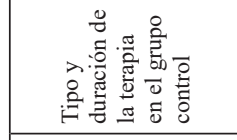 & 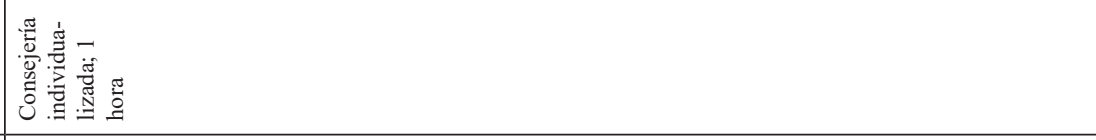 \\
\hline 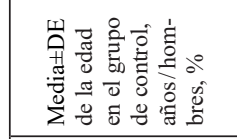 & $\begin{array}{ll}\infty & \\
\infty & \\
\text { in } & \\
\text { in } & 8 \\
n & 0 \\
\text { in } & 4 \\
\end{array}$ \\
\hline 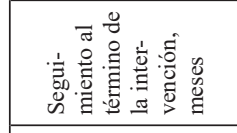 & 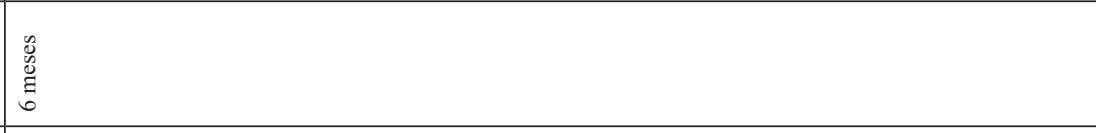 \\
\hline 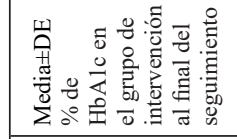 & 芫 \\
\hline 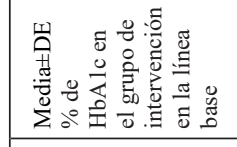 & $\tilde{z}$ \\
\hline 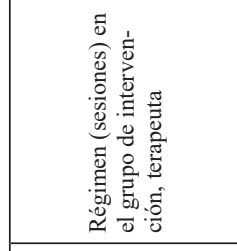 & 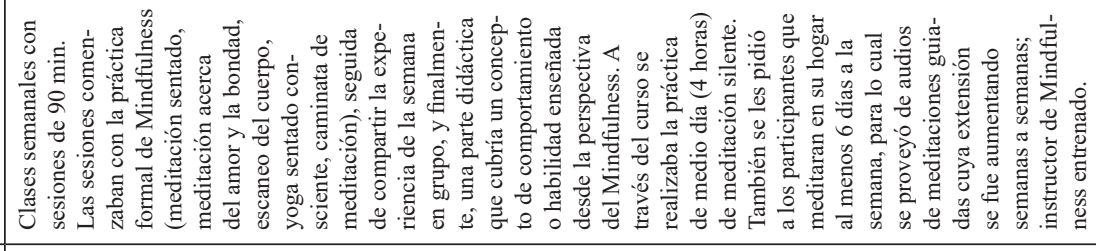 \\
\hline 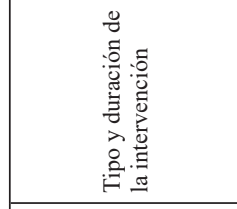 & 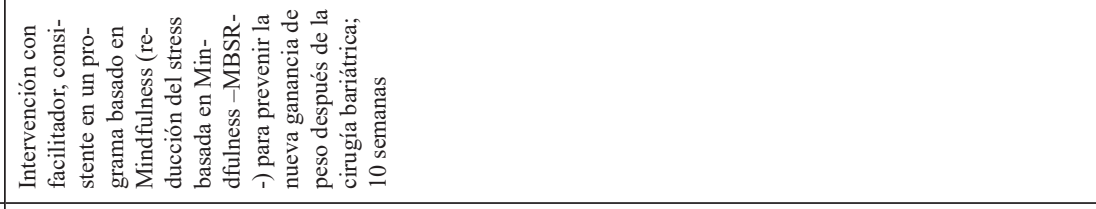 \\
\hline 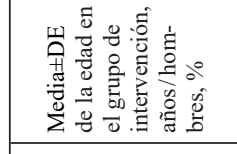 & 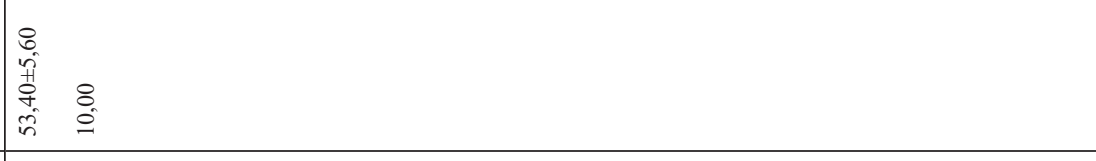 \\
\hline 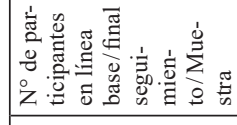 & $\underset{\infty}{\infty}$ \\
\hline 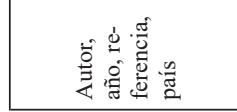 & 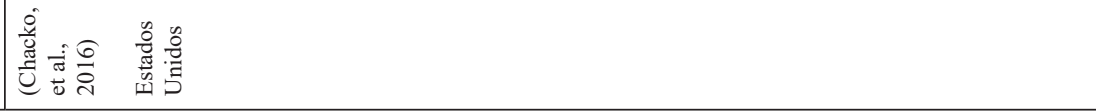 \\
\hline
\end{tabular}




\begin{tabular}{|c|c|c|c|}
\hline 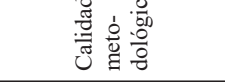 & $\frac{\pi}{4}$ & 䈥 & 愛 \\
\hline 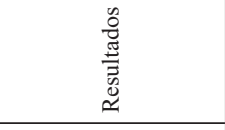 & 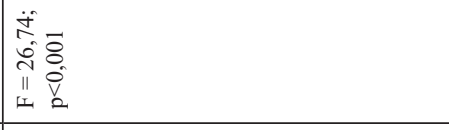 & $\tilde{z}$ & $\tilde{z}$ \\
\hline 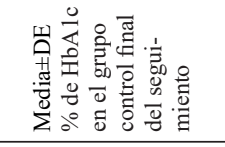 & 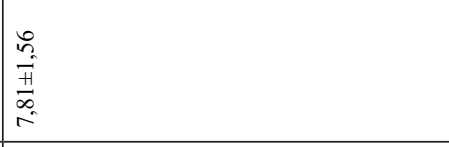 & & $\tilde{z}$ \\
\hline 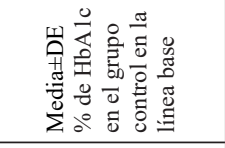 & 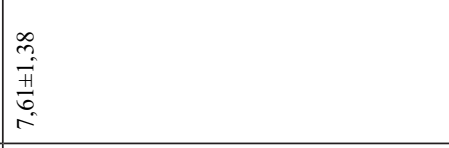 & 总 & 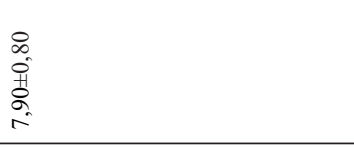 \\
\hline 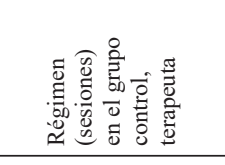 & 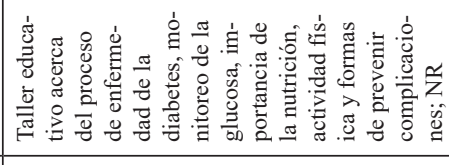 & 艺 & 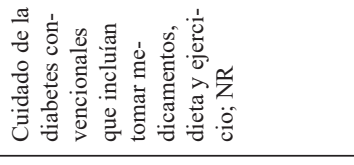 \\
\hline 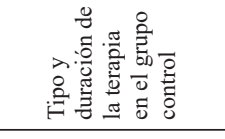 & 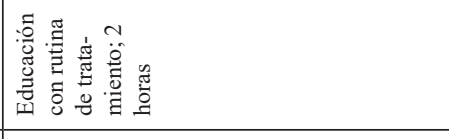 & 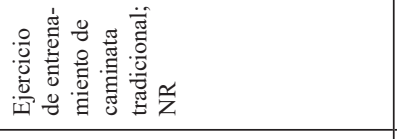 & 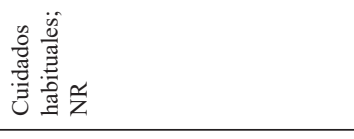 \\
\hline & 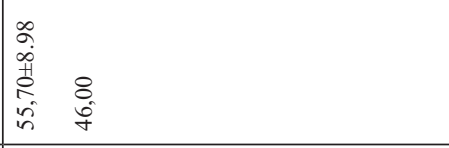 & $\begin{array}{lll} & \\
8 & & \\
0 & 0 \\
0 & 8 \\
0 & 0 \\
0\end{array}$ & 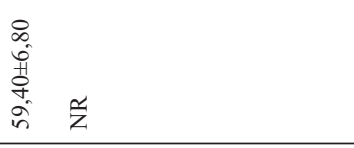 \\
\hline 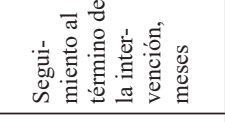 & 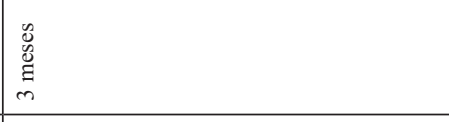 & 总 & 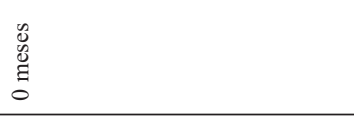 \\
\hline 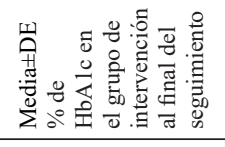 & 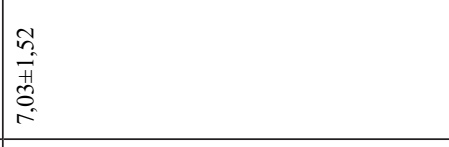 & 章 & $\tilde{z}$ \\
\hline 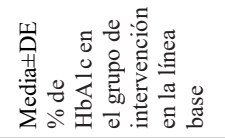 & $\mid$ & & \\
\hline 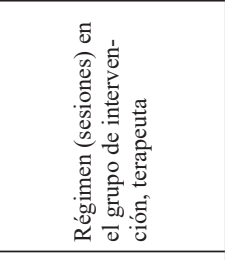 & 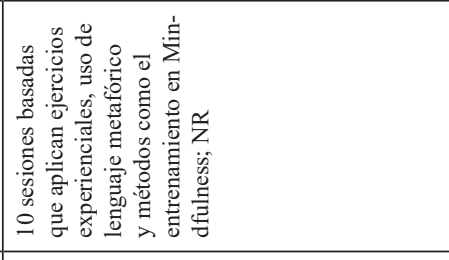 & 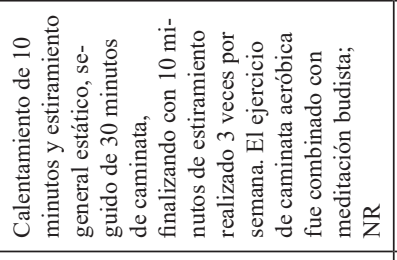 & 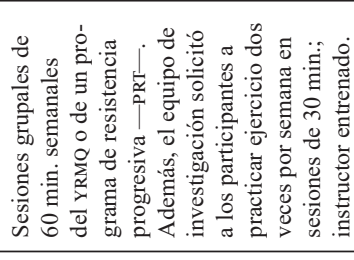 \\
\hline 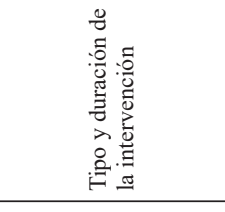 & 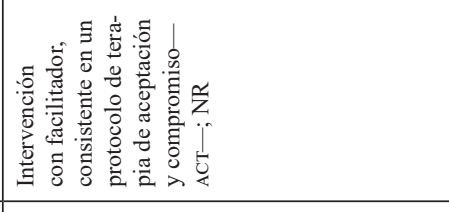 & 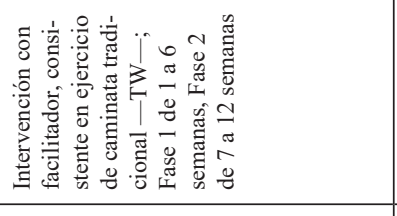 & 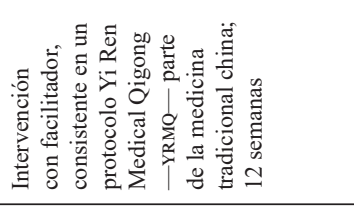 \\
\hline 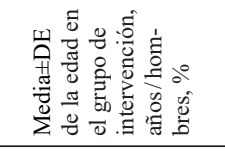 & 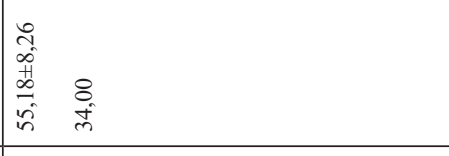 & $\begin{array}{ll}0 \\
8 \\
0 \\
0 \\
0 \\
0 \\
0 \\
0\end{array}$ & 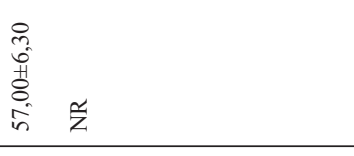 \\
\hline 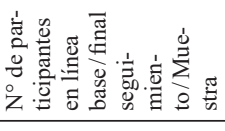 & $\begin{array}{l}\stackrel{8}{0} \\
\stackrel{0}{0} \\
\end{array}$ & 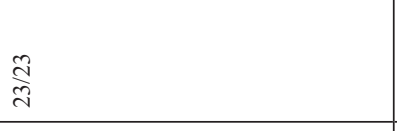 & స్ల \\
\hline 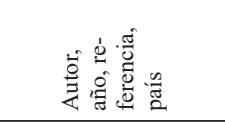 & 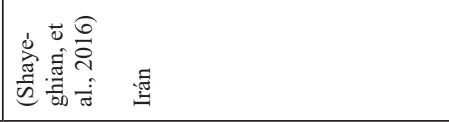 & 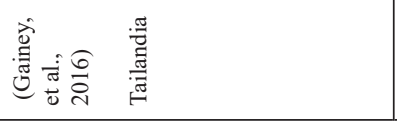 & 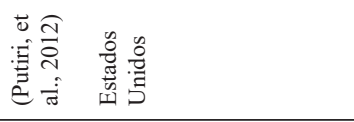 \\
\hline
\end{tabular}


Alexis González-Burboa, Soledad Luarte-Martínez, Patricia Villaseca, Camilo Manríquez, Hans Müller, Cristian Pedreros, Ángel Menéndez-Asenjo, Ángel Otero, Darío Páez y Aldo Vera-Calzaretta

\begin{tabular}{|c|c|c|}
\hline 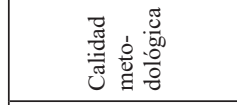 & \begin{tabular}{|l} 
离 \\
ल.
\end{tabular} & $\frac{\dddot{m}}{4}$ \\
\hline 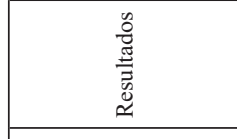 & 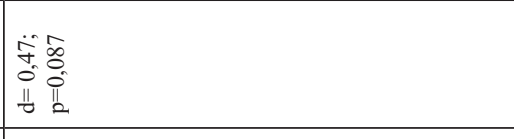 & 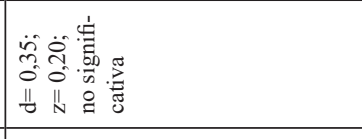 \\
\hline 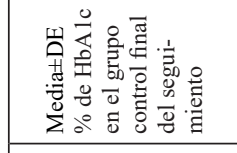 & 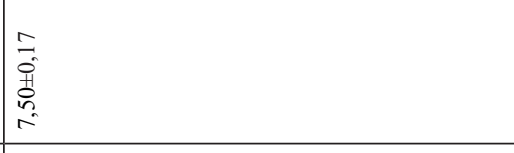 & 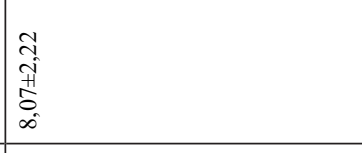 \\
\hline 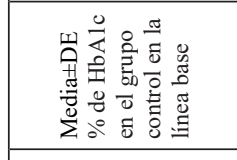 & 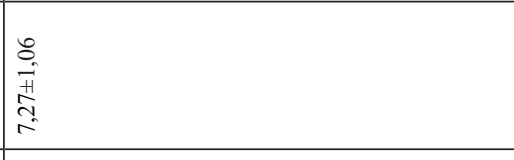 & \begin{tabular}{|l}
$\overrightarrow{\sigma_{2}}$ \\
$\vec{H}$ \\
$\vec{H}$ \\
$\infty$ \\
$\infty$ \\
\end{tabular} \\
\hline 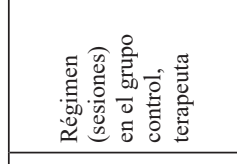 & 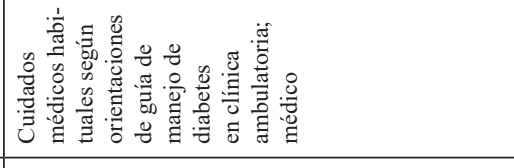 & 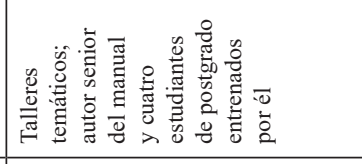 \\
\hline 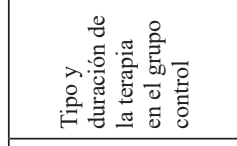 & 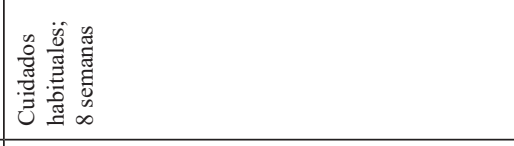 & 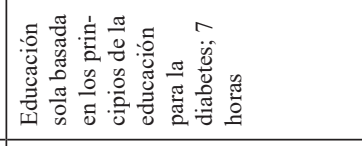 \\
\hline 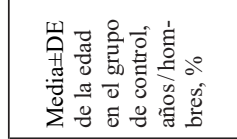 & $\begin{array}{ll}0 & \\
\infty & \\
\text { in } & \\
0 & 2 \\
0 & 2 \\
\text { in } & 0 \\
\text { in } & \infty\end{array}$ & 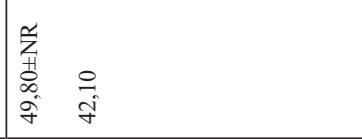 \\
\hline 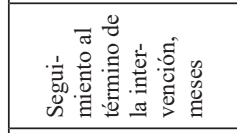 & 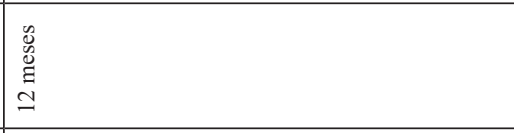 & \begin{tabular}{|l|l} 
\\
$\tilde{n}$ \\
$心$ \\
$\Xi$ \\
$m$ \\
\end{tabular} \\
\hline 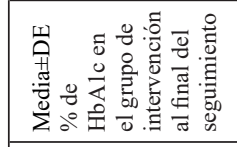 & 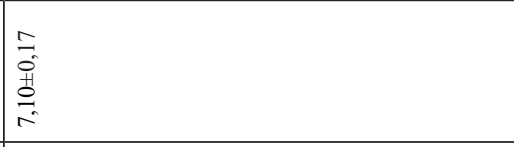 & 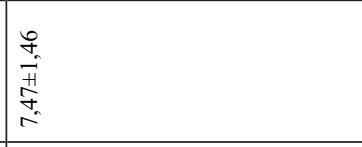 \\
\hline 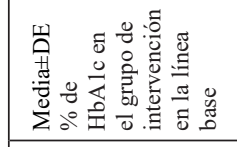 & 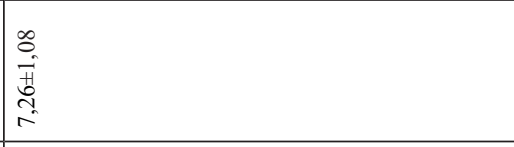 & \\
\hline 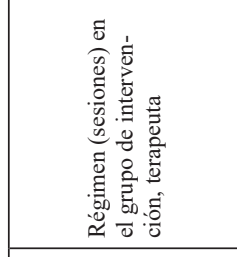 & 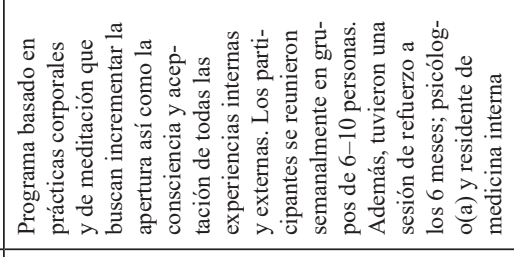 & 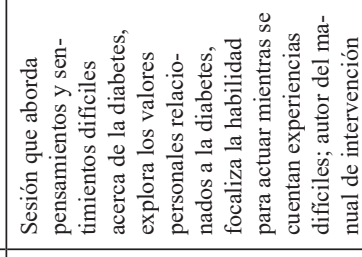 \\
\hline 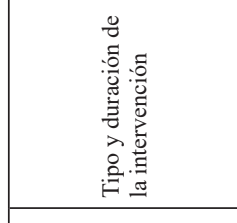 & 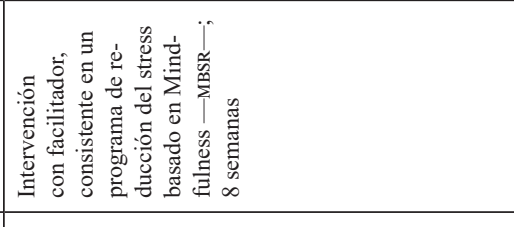 & 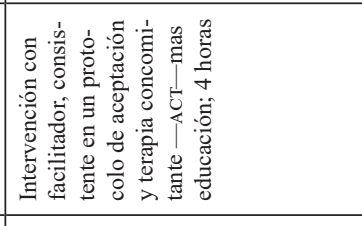 \\
\hline 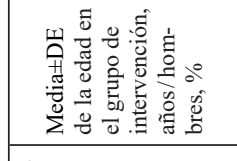 & 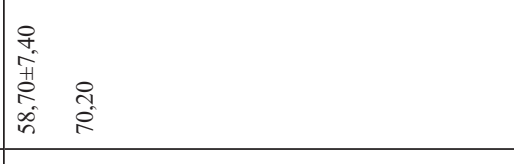 & 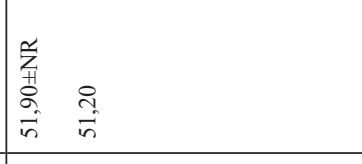 \\
\hline 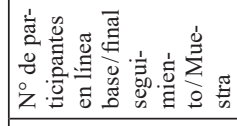 & ğ & $\frac{\Re}{\infty}$ \\
\hline 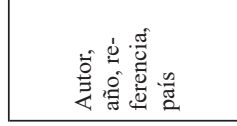 & 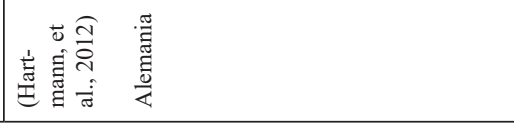 & 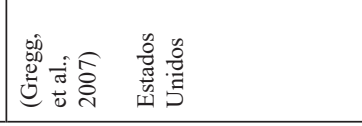 \\
\hline
\end{tabular}


vención (Armani Kian, et al., 2018; Chacko, et al., 2016; Murray, et al., 2017; Pearson, et al., 2018; Saslow, et al., 2017; Shayeghian, et al., 2016). Por otra parte, hubo dos estudios en los cuales no se observó una disminución de esta (Gregg, et al., 2007; Hartmann, et al., 2012), y dos que no reportaron resultados (Gainey, et al., 2016; Putiri, et al.,
2012). En la Tabla 1 se presentan las características de los estudios sistematizados.

Tabla 2. Otras mediciones de variables psicológicas reportadas en los estudios sorteados por año de publicación.

\begin{tabular}{|c|c|c|c|c|c|}
\hline $\begin{array}{l}\text { Autor, año, } \\
\text { referencia, país }\end{array}$ & $\begin{array}{c}\text { Media } \pm \mathrm{DE} \\
\text { en el grupo de intervención en } \\
\text { la línea base }\end{array}$ & $\begin{array}{c}\text { Media } \pm \mathrm{DE} \\
\text { en el grupo de intervención al } \\
\text { final del seguimiento }\end{array}$ & $\begin{array}{c}\text { Media } \pm \mathrm{DE} \\
\text { en el grupo control en la } \\
\text { línea base }\end{array}$ & $\begin{array}{c}\text { Media } \pm \mathrm{DE} \\
\text { en el grupo control final del } \\
\text { seguimiento }\end{array}$ & Resultados \\
\hline $\begin{array}{l}\text { (Pearson, et al., } \\
\text { 2018) } \\
\text { Australia }\end{array}$ & $\begin{array}{c}\text { DASS }^{1}-21 \text { depresión }=10,80 \\
\pm 8,90 \\
\text { DASS-21 ansiedad }=7,20 \\
\pm 6,00 \\
\text { DASS-21 stress }=10,10 \pm \\
7,20 \\
\text { PAID }^{2}=19,2 \pm 14,7\end{array}$ & $\begin{array}{c}\text { DASS-21 depresión }=6,00 \\
\pm 6,40 \\
\text { DASS-21 ansiedad }=5,60 \\
\pm 5,40 \\
\text { DASS-21 stress }=6,70 \pm 6,20 \\
\text { PAID }=11,8 \pm 14,7\end{array}$ & $\begin{array}{c}\text { DASS-21 depresión }=7,30 \\
\pm 8,70 \\
\text { DASS-21 } \\
\pm 6,70 \\
\pm 6,70 \text { and }=6,40 \\
\text { DASS-21 stress }=7,40 \pm 7,20 \\
\text { PAID }=15,2 \pm 14,6\end{array}$ & $\begin{array}{c}\text { DASS-21 depresión }=7,50 \\
\pm 8,10 \\
\text { DASS-21 } \\
\pm 7,00 \\
\text { DAsiedad }=7,10 \\
\text { DASS-21 stress }=8,70 \pm 8,20 \\
\text { PAID }=12,5 \pm 13,1\end{array}$ & $\begin{array}{c}\text { DASS-21 depresión } \\
\text { Beta }(\text { IC } 95 \%)= \\
3,42(-0,66-7,50) ; \mathrm{p}=0,10 \\
\text { DASS- } 21 \text { ansiedad } \\
\text { Beta }(\text { IC } 95 \%)= \\
0,85(-2,09-3,79) ; \mathrm{p}=0,57 \\
\text { DASS- } 21 \text { stress } \\
\text { Beta }(\text { IC } 95 \%)= \\
3,24(-0,39-6,88) ; \mathrm{p}=0,08 \\
\text { PAID } \\
\text { Beta }(\text { IC } 95 \%)= \\
3,73(-3,68-11,15) ; \mathrm{p}=0,32\end{array}$ \\
\hline $\begin{array}{l}\text { (Armani Kian, } \\
\text { et al., 2018) } \\
\text { Irán }\end{array}$ & $\begin{array}{l}\mathrm{HARS}^{3}=36,00 \pm 11,40 \\
\mathrm{HDRS}^{4}=26,50 \pm 13,30 \\
\mathrm{GHQ}^{5}-28=31,00 \pm 9,50\end{array}$ & NR & $\begin{array}{c}\text { HARS }=28,20 \pm 9,60 \\
\text { HDRS }=27,50 \pm 12,70 \\
\text { GHQ- } 28=30,90 \pm 13,20\end{array}$ & NR & $\begin{array}{c}\text { HARS } \\
\mathrm{t}=3,22 ; \text { no significativa } \\
\text { HDRS } \\
\mathrm{t}=3,22 ; \text { no significativa } \\
\text { GHQ-28 } \\
\mathrm{t}=2,56 ; \mathrm{p}=<0,001 \\
\end{array}$ \\
\hline $\begin{array}{l}\text { (Saslow, et al., } \\
\text { 2017) } \\
\text { Estados Unidos }\end{array}$ & $\begin{array}{c}\text { DDS }^{6}=1,90 \pm 0,80 \\
\text { CES' }^{7} \text {-depresión }=10,50 \pm 7,70 \\
\text { CES-D afecto positivo }= \\
10,20 \pm 2,30 \\
\text { DES }^{8} \text { afecto negativo }=2,80 \\
\pm 1,30 \\
\text { DES afecto positivo }=6,50 \\
\pm 1,10 \\
\text { SF36 vitalidad }=53,30 \pm 16,40\end{array}$ & $\begin{array}{c}\text { DDS }=1,50 \pm \mathrm{NR} \\
\text { CES-depresión }=9,9 \pm \mathrm{NR} \\
\text { CES-D afecto positivo }= \\
10,70 \pm \mathrm{NR} \\
\text { DES afecto negativo }=2,49 \\
\pm \mathrm{NR} \\
\text { DES afecto positivo }=6,90 \\
\pm \mathrm{NR} \\
\text { SF36 vitalidad }=62,50 \pm \mathrm{NR}\end{array}$ & $\begin{array}{c}\text { DDS }=2,40 \pm 1,20 \\
\text { CES-depresión }=9,80 \pm 7,40 \\
\text { CES-D afecto positivo }= \\
10,20 \pm 2,20 \\
\text { DES afecto negativo }=2,70 \\
\pm 1,40 \\
\text { DES afecto positivo }=6,20 \\
\pm 1,50 \\
\text { SF36 vitalidad }=49,20 \pm \\
20,10\end{array}$ & $\begin{array}{c}\text { DDS }=2,00 \pm \mathrm{NR} \\
\text { CES-depresión }=8,80 \pm \mathrm{NR} \\
\text { CES-D afecto positivo }= \\
17,40 \pm \mathrm{NR} \\
\text { DES afecto negativo }=2,00 \\
\pm \mathrm{NR} \\
\text { DES afecto positivo }=6,50 \\
\pm \mathrm{NR} \\
\text { SF36 vitalidad }=60,20 \pm \mathrm{NR}\end{array}$ & $\begin{array}{c}D D S \\
\text { Dif. (IC } 95 \%)= \\
0,00(-0,50-0,50) ; \mathrm{p}=0,98 \\
\text { CES-depresión } \\
\text { Dif. (IC } 95 \%)= \\
-0,40(-4,80-5,60) ; \mathrm{p}=0,88 \\
\text { CES-D afecto positivo } \\
\text { Dif. (IC } 95 \%)= \\
-6,60(-23,5-10,30) ; \mathrm{p}=0,45 \\
\text { DES afecto negativo } \\
\text { Dif. (IC } 95 \%)= \\
0,30(0,50--0,60) ; \mathrm{p}=0,49 \\
\text { DES afecto positivo } \\
\text { Dif. (IC } 95 \%)= \\
0,10(-0,90-1,10) ; \mathrm{p}=0,82 \\
\text { SF36 vitalidad } \\
\text { Dif. (IC } 95 \%)= \\
-1,8(-15,10-11,60) ; \mathrm{p}=0,80\end{array}$ \\
\hline $\begin{array}{l}\text { (Murray, et al., } \\
\text { 2017) } \\
\text { Inglaterra }\end{array}$ & $\begin{array}{c}\text { PAID }=18,20 \pm 1,30 \\
\text { HADS }^{9}=9,30 \pm 0,50 \\
\text { DMSES }^{10}=98,80 \pm 2,40 \\
\text { DTSQ }^{11}=32,20 \pm 0,60\end{array}$ & $\begin{array}{c}\text { PAID }=14,10 \pm \mathrm{NR} \\
\mathrm{HADS}=8,25 \pm \mathrm{NR} \\
\mathrm{DMSES}=101,73 \pm \mathrm{NR} \\
\mathrm{DTSQ}=33,14 \pm \mathrm{NR}\end{array}$ & $\begin{array}{c}\text { PAID }=19,80 \pm 1,30 \\
\text { HADS }=9,10 \pm 0,50 \\
\text { DMSES }=103,60 \pm 2,30 \\
\text { DTSQ }=32,20 \pm 0,60\end{array}$ & $\begin{array}{c}\text { PAID }=17,30 \pm \mathrm{NR} \\
\text { HADS }=8,50 \pm \mathrm{NR} \\
\text { DMSES }=104,98 \pm \mathrm{NR} \\
\text { DTSQ }=32,65 \pm \mathrm{NR}\end{array}$ & $\begin{array}{c}P A I D \\
\text { Dif. (IC 95\%) }= \\
-1,50(-3,90-0,90) ; \mathrm{p}=0,209 \\
H A D S \\
\text { Dif. (IC } 95 \%)= \\
-0,45(-1,68-0,78) ; \mathrm{p}=0,474 \\
D M S E S \\
\text { Dif. (IC } 95 \%)= \\
1,55(-5,74-8,84) ; \mathrm{p}=0,674 \\
D T S Q \\
\text { Dif. (IC } 95 \%)= \\
0,49(-1,18-2,15) ; \mathrm{p}=0,564\end{array}$ \\
\hline
\end{tabular}


Alexis González-Burboa, Soledad luarte-Martínez, Patricia Villaseca, Camilo Manríquez, Hans

\begin{tabular}{|c|c|c|c|c|c|}
\hline $\begin{array}{l}\text { Autor, año, } \\
\text { referencia, país }\end{array}$ & $\begin{array}{c}\text { Media } \pm \mathrm{DE} \\
\text { en el grupo de intervención en } \\
\text { la línea base }\end{array}$ & $\begin{array}{c}\text { Media } \pm \mathrm{DE} \\
\text { en el grupo de intervención al } \\
\text { final del seguimiento }\end{array}$ & $\begin{array}{c}\text { Media } \pm \mathrm{DE} \\
\text { en el grupo control en la } \\
\text { línea base }\end{array}$ & $\begin{array}{l}\text { Media } \pm \mathrm{DE} \\
\text { en el grupo control final del } \\
\text { seguimiento }\end{array}$ & Resultados \\
\hline $\begin{array}{l}\text { (Chacko, et al., } \\
\text { 2016) } \\
\text { Estados Unidos }\end{array}$ & $\begin{array}{c}\text { TFEQ }^{16} \text { - comer emocional = } \\
42,00 \pm 21.30 \\
\text { TFEQ - restricción cognitiva } \\
=65,10 \pm 19,70 \\
\text { TFEQ alimentación no con- } \\
\text { trolada }=25,50 \pm 13,00 \\
\text { BES }^{17}=8,70 \pm 5,70 \\
\text { WEL }^{18} \text { - autoeficacia en co- } \\
\text { mer }=135,40 \pm 29,40 \\
\text { SF-36 - QOL }{ }^{19} \text { física }=49,20 \\
\pm 13,00 \\
\text { SF-36 - QOL salud mental = } \\
52,50 \pm 5,20 \\
\text { IWQOL }- \text { QOL relacionada a } \\
\text { peso }=78,40 \pm 23,10 \\
\text { CES-D - depresión }=17,00 \\
\pm 4,10 \\
\text { PSS }^{20}=12,00 \pm 6,50\end{array}$ & $\begin{array}{c}\text { TFEQ - comer emocional = } \\
37,10 \pm \text { NR } \\
\text { TFEQ - restricción cognitiva } \\
=67,00 \pm \mathrm{NR} \\
\text { TFEQ - alimentación no con- } \\
\text { trolada }=28,80 \pm \mathrm{NR} \\
\mathrm{BES}=8,50 \pm \mathrm{NR} \\
\text { WEL - autoeficacia en comer } \\
=137,10 \pm \mathrm{NR} \\
\text { SF-36 - QOL física }=50,60 \\
\pm \mathrm{NR} \\
\text { SF-36 - QOL salud mental = } \\
48,90 \pm \mathrm{NR} \\
\text { IWQOL - QOL relacionada a } \\
\text { peso }=79,70 \pm \mathrm{NR} \\
\text { CES-D - depresión }=20,40 \\
\pm \text { NR } \\
\text { PSS }=15,80 \pm \mathrm{NR}\end{array}$ & $\begin{array}{c}\text { TFEQ - comer emocional = } \\
38,30 \pm 23,60 \\
\text { TFEQ - restricción cognitiva } \\
=64,50 \pm 16,90 \\
\text { TFEQ - alimentación no con- } \\
\text { trolada }=21,80 \pm 13,90 \\
\text { BES }=10,10 \pm 6,50 \\
\text { WEL - autoeficacia en comer } \\
=139,10 \pm 27,90 \\
\text { SF-36 - QOL física }=49,10 \\
\quad \pm 10,50 \\
\text { SF-36 - QOL salud mental = } \\
54,00 \pm 9,80 \\
\text { IWQOL - QOL relacionada a } \\
\text { peso }=81,80 \pm 13,80 \\
\text { CES-D - depresión }=15,10 \\
\quad \pm 4,40 \\
\text { PSS }=10,20 \pm 6,00\end{array}$ & $\begin{array}{c}\text { TFEQ - comer emocional = } \\
44,50 \pm \mathrm{NR} \\
\text { TFEQ - restricción cognitiva } \\
=56,30 \pm \mathrm{NR} \\
\text { TFEQ - alimentación no con- } \\
\text { trolada }=25,20 \pm \mathrm{NR} \\
\mathrm{BES}=8,40 \pm \mathrm{NR} \\
\text { WEL - autoeficacia en comer } \\
=136,30 \pm \mathrm{NR} \\
\text { SF-36 - QOL física }=48,60 \\
\quad \pm \mathrm{NR} \\
\text { SF-36 - QOL salud mental = } \\
56,10 \pm \mathrm{NR} \\
\text { IWQOL - QOL relacionada a } \\
\text { peso }=82,90 \pm \mathrm{NR} \\
\text { CES-D - depresión }=17,40 \\
\pm \mathrm{NR} \\
\text { PSS }=9,60 \pm \mathrm{NR}\end{array}$ & 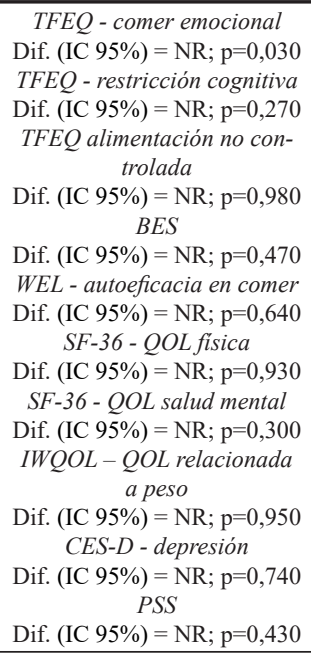 \\
\hline $\begin{array}{c}\text { (Shayeghian, } \\
\text { et al., 2016) } \\
\text { Irán }\end{array}$ & $\mathrm{SDSCA}^{21}=52,18 \pm 16,14$ & $\mathrm{SDSCA}=62,56 \pm 15,17$ & $\mathrm{SDSCA}=53,94 \pm 15,57$ & $\mathrm{SDSCA}=54,08 \pm 17,00$ & $\begin{array}{c}S D S C A \\
\mathrm{~F}=76,75 ; \mathrm{p}<0,001\end{array}$ \\
\hline $\begin{array}{l}\text { (Gainey, et al., } \\
\text { 2016) } \\
\text { Tailandia }\end{array}$ & NR & NR & NR & NR & NR \\
\hline $\begin{array}{l}\text { (Putiri, et al., } \\
\text { 2012) } \\
\text { Estados Uni- } \\
\quad \text { dos }\end{array}$ & $\begin{array}{c}\mathrm{PSS}=16,90 \pm 6,70 \\
\mathrm{BDI}=7,40 \pm 8,80\end{array}$ & NR & $\begin{array}{c}\mathrm{PSS}=14,40 \pm 5,50 \\
\mathrm{BDI}=5,00 \pm 3,10\end{array}$ & NR & $\begin{array}{c}\text { Intervención } \\
P S S \\
\text { Baja del score de } 29.3 \% \text {; } \\
\mathrm{p}=0,050 \\
B D I \\
\text { Baja del score de } 14.3 \% \text {; } \\
\text { no significativo } \\
\text { Control } \\
P S S \\
\text { Baja del score de } 2,8 \% ; \\
\text { no significativo } \\
B D I \\
\text { Aumento del score de } 2 \% ; \\
\text { no significativo }\end{array}$ \\
\hline $\begin{array}{l}\text { (Hartmann, et } \\
\text { al., 2012) } \\
\text { Alemania }\end{array}$ & $\begin{array}{l}\text { SF-12 escala compuesta sa- } \\
\text { lud mental }=45,60 \pm 10,3 \\
\text { SF-12 escala compuesta sa- } \\
\text { lud física }=37,90 \pm 10,3 \\
\text { PHQ-9 score de depresión }= \\
6,40 \pm 4,90 \\
\text { PHQ score de stress }=6,40 \\
\pm 3,60\end{array}$ & $\begin{array}{l}\text { SF-12 escala compuesta sa- } \\
\text { lud mental }=49,20 \pm 1,69 \\
\text { SF-12 escala compuesta sa- } \\
\text { lud física }=39,20 \pm 1,11 \\
\text { PHQ-9 score de depresión }= \\
5,00 \pm 0,55 \\
\text { PHQ score de stress }=4,60 \\
\pm 0,48\end{array}$ & $\begin{array}{l}\text { SF-12 escala compuesta sa- } \\
\text { lud mental }=43,90 \pm 10,80 \\
\text { SF-12 escala compuesta sa- } \\
\text { lud física }=39,60 \pm 10,80 \\
\text { PHQ-9 score de depresión }= \\
5,70 \pm 4,30 \\
\text { PHQ score de stress }=6,00 \\
\pm 3,70\end{array}$ & $\begin{array}{l}\text { SF-12 escala compuesta sa- } \\
\text { lud mental }=43,50 \pm 1,69 \\
\text { SF-12 escala compuesta sa- } \\
\text { lud física }=40,30 \pm 1,12 \\
\text { PHQ-9 score de depresión }= \\
7,30 \pm 0,57 \\
\text { PHQ score de stress }=6,20 \\
\pm 0,52\end{array}$ & $\begin{array}{c}\text { SF-12 escala compuesta salud } \\
\text { mental } \\
\mathrm{d}=0,65 ; \mathrm{p}=0,018 \\
\text { SF-12 escala compuesta salud } \\
\text { física } \\
\mathrm{d}=0,19 ; \mathrm{p}=0,480 \\
\text { PHQ-9 score de depresión } \\
\mathrm{d}=0,79 ; \mathrm{p}=0,005 \\
\text { PHQ score de stress } \\
\mathrm{d}=0,64 ; \mathrm{p}=0,0023\end{array}$ \\
\hline $\begin{array}{l}\text { (Gregg, et al., } \\
\text { 2007) } \\
\text { Estados Uni- } \\
\quad \text { dos }\end{array}$ & $\begin{array}{l}\text { Autogestión }=7,49 \pm 5,87 \\
\mathrm{AADQ}^{27}=46,23 \pm 16,97 \\
\mathrm{DCP}^{28} \text { comprensión y sati- } \\
\text { sfacción tratamiento }= \\
49,56 \pm 16,66\end{array}$ & $\begin{array}{c}\text { Autogestión }=13,58 \pm 5,56 \\
\text { AADQ }=54,10 \pm 15,24 \\
\text { DCP comprensión y sati- } \\
\text { sfacción tratamiento }=55,53 \\
\quad \pm 15,73\end{array}$ & $\begin{array}{l}\text { Autogestión }=10,28 \pm 5,99 \\
\text { AADQ }=50,58 \pm 16,12 \\
\text { DCP comprensión y sati- } \\
\text { sfacción tratamiento }=45,61 \\
\quad \pm 16,88\end{array}$ & $\begin{array}{l}\text { Autogestión }=12,41 \pm 5,21 \\
\text { AADQ }=48,43 \pm 14,86 \\
\text { DCP comprensión y sati- } \\
\text { sfacción tratamiento }=48,13 \\
\pm 17,37\end{array}$ & $\begin{array}{c}\text { Autogestión } \\
\mathrm{d}=0,68 ; \mathrm{z}=-2,40, \mathrm{p}<0,05 \\
A A D Q \\
\mathrm{~d}=0,78 ; \mathrm{z}=-3,22, \mathrm{p}<0,01 \\
\text { DCP comprensión y satisfac- } \\
\text { ción tratamiento } \\
\mathrm{d}=0,38 ; \mathrm{z}=-1,08, \text { no signifi- } \\
\text { cativo }\end{array}$ \\
\hline
\end{tabular}


Al analizar distintas variables de tipo psicológicas incluidas en estos estudios, se encontró que en solo cinco estudios las intervenciones tuvieron un efecto en la mejoría de síntomas de depresión (Hartmann, et al., 2012), la percepción de salud general (Armani Kian, et al., 2018), el consumo de alimentos con base emocional (Chacko, et al., 2016), el estrés percibido (Hartmann, et al., 2012; Putiri, et al., 2012), la aceptación de la diabetes (Gregg, et al., 2007), la autogestión de la enfermedad (Gregg, et al., 2007) y el autocuidado (Shayeghian, et al., 2016). El resto de las variables consideradas en los estudios no reportaron diferencias estadísticamente significativas (Tabla 2).

\section{Calidad de los estudios}

Las evaluaciones independientes de la calidad metodológica de los artículos incluidos en la síntesis cualitativa, logró un nivel de acuerdo considerable $(\kappa=0,686 ; p<0,001)$. De esta forma, seis artículos fueron catalogados con una alta calidad (Chacko, et al., 2016; Gregg, et al., 2007; Murray, et al., 2017; Pearson, et al., 2018; Saslow, et al., 2017; Shayeghian, et al., 2016) y cuatro con una baja calidad (Armani Kian, et al., 2018; Gainey, et al., 2016; Hartmann, et al., 2012; Putiri, et al., 2012).
Todos los estudios fueron aleatorizados, no obstante, cuatro de estos realizaron doble enmascaramiento (Chacko, et al., 2016; Gregg, et al., 2007; Pearson, et al., 2018; Saslow, et al., 2017), y seis describieron los abandonos y exclusiones del estudio (Gainey, et al., 2016; Gregg, et al., 2007; Murray, et al., 2017; Pearson, et al., 2018; Saslow, et al., 2017; Shayeghian, et al., 2016).

\section{Síntesis cuantitativa de los estudios}

De los 10 estudios seleccionados para la síntesis cualitativa, finalmente los resultados de 7 trabajos fueron considerados en la integración metanalítica (Gainey, et al., 2016; Gregg, et al., 2007; Hartmann, et al., 2012; Murray, et al., 2017; Pearson, et al., 2018; Saslow, et al., 2017; Shayeghian, et al., 2016). En esta etapa, se descartaron 3 estudios, ya que, no reportaron mediciones post intervención (Armani Kian, et al., 2018; Chacko, et al., 2016; Putiri, et al., 2012).

En el cálculo de la diferencia de medias, la heterogeneidad fue moderada $\left(\mathrm{Chi}^{2}=11,84, \mathrm{df}=6(\mathrm{p}=0.07) ; \mathrm{I}^{2}=49 \%\right)$. El modelo de efectos fijos mostró un efecto global de -0.25 $(-0.27--0.23)$, mientras que para el de efectos aleatorios fue de $-0.29(-0.37--0.21)$.

Por otra parte, la diferencia de medias estandarizadas mostró una considerable heterogeneidad $\left(\mathrm{Chi}^{2}=125,94\right.$, $\left.\mathrm{df}=6(\mathrm{p}<0.00001) ; \mathrm{I}^{2}=95 \%\right)$, obteniéndose un efecto glo-

Tabla 3. Integración metanalítica preliminar del efecto del Mindfulness sobre la HbAlc.

\begin{tabular}{|c|c|c|c|c|c|c|}
\hline Autor & $\begin{array}{l}\text { Dif. Media } \\
\text { (IC 95\%) }\end{array}$ & $\begin{array}{l}\text { Peso relativo } \\
\text { MEF }^{1}, \%\end{array}$ & $\begin{array}{l}\text { Peso relativo } \\
\text { MEA }^{2}, \%\end{array}$ & $\begin{array}{l}\text { Dif. Media } \\
\text { Estandarizada } \\
\text { (IC 95\%) }\end{array}$ & $\begin{array}{l}\text { Peso relativo } \\
\text { MEF, \% }\end{array}$ & $\begin{array}{l}\text { Peso relativo } \\
\text { MEA, \% }\end{array}$ \\
\hline (Pearson, et al., 2018) & $0,01(-0,73-0,75)$ & 0,1 & 1,1 & $0.01(-0,52-0,53)$ & 11,1 & 14,6 \\
\hline (Saslow, et al., 2017) & $-0,60(-0,93--0,27)$ & 0,4 & 5,1 & $-1,66(-2,75--0,58)$ & 2,6 & 12,3 \\
\hline (Murray, et al., 2017) & $-0,24(-0,26--0,22)$ & 87,8 & 51,0 & $-2,39(-2,68--2,11)$ & 36,4 & 15,2 \\
\hline (Shayeghian, et al., 2016) & $-0,78(-1,38--0,18)$ & 0,1 & 1,6 & $-0.50(-0,90--0,10)$ & 19,1 & 14,9 \\
\hline (Gainey, et al., 2016) & $-0,51(-2,58-1,56)$ & 0,0 & 0,1 & $-0.20(-1,02-0,63)$ & 4,5 & 13,5 \\
\hline (Hartmann, et al., 2012) & $-0,30(-0,36--0,24)$ & 11,5 & 40,3 & $-1.97(-2,47--1,47)$ & 12,3 & 14,7 \\
\hline (Gregg, et al., 2007) & $-0,60(-1,48-0,28)$ & 0,1 & 0,8 & $-0.32(-0,79-0,14)$ & 14,1 & 14,8 \\
\hline Total (IC 95\%) & & $\begin{array}{c}-0,25(-0,27- \\
-0,23) \\
\end{array}$ & $\begin{array}{c}-0,29(-0,37- \\
-0,21) \\
\end{array}$ & & $\begin{array}{c}-1,30(-1,48- \\
-1,13)\end{array}$ & $\begin{array}{c}-1,01(-1,85- \\
-0,16) \\
\end{array}$ \\
\hline Efecto global & & $\begin{array}{l}Z=23,68 \\
p<0,00001\end{array}$ & $\begin{array}{c}\mathrm{Z}=7,31 \\
\mathrm{p}<0,00001\end{array}$ & & $\begin{array}{c}Z=14,68 \\
p<0,00001\end{array}$ & $\begin{array}{c}Z=2,32 \\
p=0,02\end{array}$ \\
\hline Heterogeneidad & & $\begin{array}{c}\mathrm{Chi}^{2}=11,84 \\
\mathrm{df}=6(\mathrm{p}=0,07) \\
\mathrm{I}^{2}=49 \%\end{array}$ & $\begin{array}{c}\mathrm{Tau}^{2}=0,00 \\
\mathrm{Chi}^{2}=11,84 \\
\mathrm{df}=6(\mathrm{p}=0,07) \\
\mathrm{I}^{2}=49 \%\end{array}$ & & $\begin{array}{c}\mathrm{Chi}^{2}=125,94 \\
\mathrm{df}=6 \\
(\mathrm{p}<0,00001) \\
\mathrm{I}^{2}=95 \%\end{array}$ & $\begin{array}{c}\mathrm{Tau}^{2}=1,21 ; \\
\mathrm{Chi}^{2}=125,94, \\
\mathrm{df}=6 \\
(\mathrm{p}<0,00001) \\
\mathrm{I}^{2}=95 \%\end{array}$ \\
\hline
\end{tabular}

${ }^{1}$ MEF: Modelo de efectos fijos; ${ }^{2}$ MEA: Modelo de efectos aleatorios 
Alexis González-Burboa, Soledad Luarte-Martínez, Patricia Villaseca, Camilo Manríquez, Hans

Müller, Cristian Pedreros, Ángel Menéndez-Asenjo, Ángel Otero, Darío Páez y Aldo Vera-Calzaretta

Figura 2. Efectividad de intervenciones basadas en Mindfulness sobre el control glicémico (\% HbAlc)

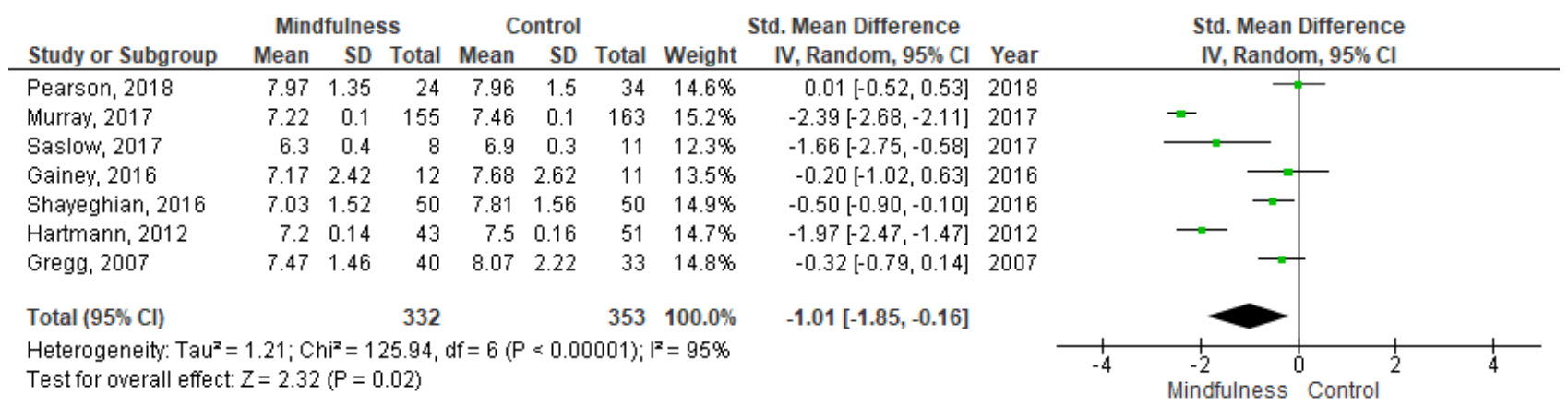

Tabla 4. Diferencias de medias estandarizadas del modelo de efectos aleatorios, por subgrupo.

\begin{tabular}{|c|c|c|c|c|c|}
\hline Subgrupos & Estudios incluidos & $\begin{array}{l}\text { Dif. Media } \\
\text { Estandarizada } \\
\quad(\text { IC } 95 \%)\end{array}$ & Efecto global & Heterogeneidad & $\begin{array}{l}\text { Diferencia entre } \\
\text { subgrupos }\end{array}$ \\
\hline \multicolumn{6}{|c|}{ NÚMERO DE PARTICIPANTES EN EL GRUPO DE INTERVENCIÓN } \\
\hline$<30$ casos & $\begin{array}{c}\text { (Gainey, et al., 2016; Pearson, } \\
\text { et al., 2018; Saslow, et al., } \\
\text { 2017) }\end{array}$ & $-0,51(-1,39-0,36)$ & $Z=1,15(p=0,25)$ & $\begin{array}{c}\mathrm{Tau}^{2}=0,43 ; \mathrm{Chi}^{2}=7,41 \\
\mathrm{df}=2(\mathrm{p}=0,02) ; \mathrm{I}^{2}=73 \%\end{array}$ & $\begin{array}{l}\mathrm{Chi}^{2}=1,22, \mathrm{df}=1(\mathrm{p} \\
=0,27), \mathrm{I}^{2}=18,1 \%\end{array}$ \\
\hline 30 o más casos & $\begin{array}{l}\text { (Gregg, et al., 2007; } \\
\text { Hartmann, et al., 2012; } \\
\text { Murray, et al., 2017; } \\
\text { Shayeghian, et al., 2016) }\end{array}$ & $-1,30(-2,39--0,21)$ & $\mathrm{Z}=2,34(\mathrm{p}=0,02)$ & $\begin{array}{c}\mathrm{Tau}^{2}=1,19 ; \mathrm{Chi}^{2}=88,95 \\
\mathrm{df}=3 \\
(\mathrm{p}<0,00001) ; \mathrm{I}^{2}=97 \%\end{array}$ & \\
\hline \multicolumn{6}{|c|}{ PROPORCIÓN DE HOMBRES / MUJERES } \\
\hline $\begin{array}{l}>\text { proporción de } \\
\text { hombres }\end{array}$ & $\begin{array}{l}\text { (Gregg, et al., 2007; } \\
\text { Hartmann, et al., 2012; } \\
\text { Murray, et al., 2017) }\end{array}$ & $-1,57(-2,82--0,32)$ & $\mathrm{Z}=2,46(\mathrm{p}=0,01)$ & $\begin{array}{c}\mathrm{Tau}^{2}=1,17 ; \mathrm{Chi}^{2}=55,49 \\
\mathrm{df}=2(\mathrm{p}<0,00001) \\
\mathrm{I}^{2}=96 \%\end{array}$ & $\begin{array}{c}\mathrm{Chi}^{2}=3,79, \mathrm{df}=1 \\
(\mathrm{p}=0,05), \\
\mathrm{I}^{2}=73,6 \%\end{array}$ \\
\hline $\begin{array}{l}>\text { proporción de } \\
\text { mujeres }\end{array}$ & $\begin{array}{c}\text { (Gainey, et al., 2016; Pearson, } \\
\text { et al., 2018; Shayeghian, et } \\
\text { al., 2016) }\end{array}$ & $-0,29(-0,62-0,05)$ & $Z=1,68(p=0,09)$ & $\begin{array}{c}\mathrm{Tau}^{2}=0,01 ; \mathrm{Chi}^{2}=2,38 \\
\mathrm{df}=2(\mathrm{p}=0,30) ; \mathrm{I}^{2}=16 \%\end{array}$ & \\
\hline \multicolumn{6}{|c|}{ TIPO DE INTERVENCIÓN } \\
\hline $\begin{array}{l}\text { Individual auto- } \\
\text { aplicada }\end{array}$ & $\begin{array}{c}\text { (Murray, et al., 2017; Pearson, } \\
\text { et al., 2018; Saslow, et al., } \\
\text { 2017) }\end{array}$ & $-1,35(-3,10-0,40)$ & $\mathrm{Z}=1,51(\mathrm{p}=0,13)$ & $\begin{array}{c}\mathrm{Tau}^{2}=2,26 ; \mathrm{Chi}^{2}=62,22 \\
\mathrm{df}=2(\mathrm{p}<0,00001) \\
\mathrm{I}^{2}=97 \%\end{array}$ & $\begin{array}{l}\mathrm{Chi}^{2}=0,35, \mathrm{df}=1 \\
(\mathrm{p}=0,55), \mathrm{I}^{2}=0 \%\end{array}$ \\
\hline $\begin{array}{l}\text { Grupal con } \\
\text { facilitador }\end{array}$ & $\begin{array}{c}\text { (Gainey, et al., 2016; Gregg, } \\
\text { et al., 2007; Hartmann, et } \\
\text { al., 2012; Shayeghian, et al., } \\
\text { 2016) }\end{array}$ & $-0,76(-1,57-0,04)$ & $\mathrm{Z}=1,86(\mathrm{p}=0,06)$ & $\begin{array}{c}\mathrm{Tau}^{2}=0,59 ; \mathrm{Chi}^{2}=29,47 \\
\mathrm{df}=3(\mathrm{p}<0,00001) \\
\mathrm{I}^{2}=90 \%\end{array}$ & \\
\hline \multicolumn{6}{|c|}{ DURACIÓN DE LA INTERVENCIÓN } \\
\hline $\begin{array}{l}8 \text { o menos } \\
\text { semanas }\end{array}$ & $\begin{array}{l}\text { (Gregg, et al., 2007; } \\
\text { Hartmann, et al., 2012; } \\
\text { Pearson, et al., 2018) }\end{array}$ & $-0,76(-1,94-0,42)$ & $\mathrm{Z}=1,26(\mathrm{p}=0,21)$ & $\begin{array}{c}\mathrm{Tau}^{2}=1,03 ; \mathrm{Chi}^{2}=34,36 \\
\mathrm{df}=2 \\
(\mathrm{p}<0,00001) ; \mathrm{I}^{2}=94 \%\end{array}$ & $\begin{array}{l}\mathrm{Chi}^{2}=0,51, \mathrm{df}=1 \\
(\mathrm{p}=0,47), \mathrm{I}^{2}=0 \%\end{array}$ \\
\hline$>8$ semanas & $\begin{array}{c}\text { (Gainey, et al., 2016; Murray, } \\
\text { et al., 2017; Saslow, et al., } \\
\text { 2017) }\end{array}$ & $-1,44(-2,90-0,01)$ & $\mathrm{Z}=1,95(\mathrm{p}=0,05)$ & $\begin{array}{c}\mathrm{Tau}^{2}=1,49 ; \mathrm{Chi}^{2}=25,28 \\
\mathrm{df}=2 \\
(\mathrm{p}<0,00001) ; \mathrm{I}^{2}=92 \%\end{array}$ & \\
\hline
\end{tabular}




\begin{tabular}{|c|c|c|c|c|c|}
\hline Subgrupos & Estudios incluidos & $\begin{array}{l}\text { Dif. Media } \\
\text { Estandarizada } \\
(\text { IC } 95 \%)\end{array}$ & Efecto global & Heterogeneidad & $\begin{array}{l}\text { Diferencia entre } \\
\text { subgrupos }\end{array}$ \\
\hline \multicolumn{6}{|c|}{ DURACIÓN DEL SEGUIMIENTO } \\
\hline$<12$ meses & $\begin{array}{l}\text { (Gainey, et al., 2016; Gregg, } \\
\text { et al., 2007; Pearson, et al., } \\
\text { 2018; Saslow, et al., 2017; } \\
\text { Shayeghian, et al., 2016) }\end{array}$ & $-0,40(-0,78--0,03)$ & $\mathrm{Z}=2,12(\mathrm{p}=0,03)$ & $\begin{array}{c}\mathrm{Tau}^{2}=0,09 ; \mathrm{Chi}^{2}=8,07 \\
\mathrm{df}=4(\mathrm{p}=0,09) ; \mathrm{I}^{2}=50 \%\end{array}$ & $\begin{array}{c}\mathrm{Chi}^{2}=42,26, \mathrm{df}=1 \\
(\mathrm{p}<0,00001), \\
\mathrm{I}^{2}=97,6 \%\end{array}$ \\
\hline 12 meses & $\begin{array}{l}\text { (Hartmann, et al., 2012; } \\
\text { Murray, et al., 2017) }\end{array}$ & $-2,23(-2,64--1,82)$ & $\begin{array}{c}Z=10,77 \\
(p<0,00001)\end{array}$ & $\begin{array}{c}\mathrm{Tau}^{2}=0,05 ; \mathrm{Chi}^{2}=2,11 \\
\mathrm{df}=1(\mathrm{p}=0,15) ; \mathrm{I}^{2}=53 \%\end{array}$ & \\
\hline
\end{tabular}

bal de -1.30 (-1.48- -1.13) para el modelo de efectos fijos, y un efecto global de $-1.01(-1.85--0.16)$ para el modelo de efectos aleatorios (Tabla 3). En la figura 2, se presenta el forrest plot de la diferencia de medias estandarizadas (modelo de efectos aleatorios).

El análisis por subgrupos mostró efectos estadísticamente significativos en aquellos estudios que tenían un tamaño muestral de 30 o más casos $(\mathrm{p}=0.02)$, una mayor proporción de hombres $(\mathrm{p}=0.01)$ y un seguimiento menor o igual a 12 meses $(\mathrm{p}=0.03)$, así como, de 12 meses $(\mathrm{p}<0.00001)$. Los otros subgrupos no mostraron efectos estadísticamente significativos.

\section{Discusión}

Se identificaron 10 estudios experimentales aleatorizados que evaluaron el efecto de intervenciones que incorporaron el Mindfulness como parte de su diseño sobre los niveles de HbA1c en personas con DM2. De estos, seis reportaron una diminución estadísticamente significativa de los niveles de $\mathrm{HbA} 1 \mathrm{c}$ en el grupo de intervención al comparar las mediciones pre y post test.

Por otra parte, al analizar el efecto de las intervenciones que incluían Mindfulness sobre variables de tipo psicológicas, se encontró que solo cinco trabajos reportaron resultados significativos. A su vez, cabe observar que estas variables, no fueron las mismas para todos los estudios, encontrándose una notoria heterogeneidad (ej. depresión, ansiedad, salud general, estrés psicológico, etc.). Esta situación impidió llevar a cabo una posterior integración metanalítica en este ámbito.

No obstante lo anterior, por una parte, se alienta la idea de utilizar este tipo de intervenciones como coadyuvante del tratamiento médico de la DM2 y, por otra, se hace necesario seguir profundizando en la relación de las intervenciones que incluyen Mindfulness con la modificación de distintas variables psicológicas en estas personas.
Respecto a los estudios incorporados en la integración metanalítica se encontró que la utilización del Mindfulness tuvo un efecto sobre la disminución de los niveles de HbA1c, no obstante, se observó una considerable heterogeneidad en el análisis $\left(I^{2}=95 \%\right)$. Esta, podría atribuirse a que las intervenciones implementadas presentaron diferencias en cuanto a su estructura, duración, forma de implementación, número y sexo de los participantes, tiempo de seguimiento, entre otras.

En específico, una primera variable que se consideró para entender dicha heterogeneidad fue el tamaño de las muestras en los estudios. Se observó que aquellos estudios que tenían menos de 30 participantes en la intervención, no reportaron un efecto estadísticamente significativo (Gainey, et al., 2016; Pearson, et al., 2018; Saslow, et al., 2017), en contraposición de aquellos que tuvieron 30 o más participantes (Gregg, et al., 2007; Hartmann, et al., 2012; Murray, et al., 2017; Shayeghian, et al., 2016). En este último grupo, hubo un leve aumento del tamaño del efecto en relación al de la totalidad de los estudios (-1,30 versus $-1,01)$.

Sumado a lo anterior, otra variable de agrupación considerada para el análisis fue la proporción de hombres y mujeres que componían el grupo de intervención. En concreto, aquellos estudios cuyos participantes fueron en su mayoría hombres (Gregg, et al., 2007; Hartmann, et al., 2012; Murray, et al., 2017), presentaron un aumento en el tamaño del efecto sobre la reducción de los niveles de $\mathrm{HbAlc}$ en relación al efecto total $(-1,57$ versus $-1,01)$. Cuando la proporción de mujeres fue mayor, no se observaron diferencias estadísticamente significativas (Gainey, et al., 2016; Pearson, et al., 2018; Shayeghian, et al., 2016). Esto podría hacer necesario considerar la perspectiva de enfoque de género para adaptar las intervenciones de forma de hacerlas más efectivas y acordes al público objetivo.

En relación al tiempo de seguimiento una vez finalizada la intervención, se encontró un aumento significativo del 
efecto en la reducción de la $\mathrm{HbAlc}$ en aquellos estudios que tuvieron un seguimiento de un año $(-2,23$ versus $-1,01)$ (Hartmann, et al., 2012; Murray, et al., 2017).

Respecto a la modalidad de las intervenciones (grupal o auto-aplicada) así como su duración, no se encontró un efecto estadísticamente significativo en la reducción de la $\mathrm{HbA1c}$.

Finalmente, dentro de las limitaciones de este trabajo se plantea la alta heterogeneidad de los estudios, lo cual, podría sobreestimar los efectos encontrados en la integración metanalítica. Por otra parte, lo incipiente del desarrollo del conocimiento en el área al momento de realizar este trabajo podría aumentar el riesgo de presentar sesgo de publicación.

\section{Conclusión}

La implementación de intervenciones basadas en Mindfulness tendría un efecto sobre la reducción de los niveles de HbA1c de los pacientes con DM2. Pese a que no existe un consenso respecto a las técnicas ni los aspectos logísticos de la implementación de estas intervenciones, sería recomendable su uso como apoyo al trabajo de los equipos de salud en el manejo de personas diabéticas. No obstante, dado el número de estudios considerados y las limitaciones de este trabajo, es necesario seguir profundizando en la utilización de este tipo de estrategia de intervención.

\section{Referencias}

Alam, R., Sturt, J., Lall, R. \& Winkley, K. (2009). An updated meta-analysis to assess the effectiveness of psychological interventions delivered by psychological specialists and generalist clinicians on glycaemic control and on psychological status. Patient Education and Counseling, 75(1), 25-36.

Armani Kian, A., Vahdani, B., Noorbala, A. A., Nejatisafa, A., Arbabi, M., Zenoozian, S. \& Nakhjavani, M. (2018). The Impact of Mindfulness-Based Stress Reduction on Emotional Wellbeing and Glycemic Control of Patients with Type 2 Diabetes Mellitus. Journal of Diabetes Research, 2018.

Blot, W. J., McLaughlin, J. K., Winn, D. M., Austin, D. F., Greenberg, R. S., Preston-Martin, S., ... Fraumeni, J. F. (1988). Smoking and drinking in relation to oral and pharyngeal cancer. Cancer Research, 48(11), 3282-3287.

Bodenheimer, T., Wagner, E. H. \& Grumbach, K. (2002). Improving primary care for patients with chronic illness. Journal of the American Medical Association, 288(14), 1775-1779.

Bohlmeijer, E., Prenger, R., Taal, E. \& Cuijpers, P. (2010). The effects of mindfulness-based stress reduction therapy on mental health of adults with a chronic medical disease: a meta-analysis. Journal of Psychosomatic Research, 68(6), 539-544.
Chacko, S. A., Yeh, G. Y., Davis, R. B. \& Wee, C.C. (2016). A mindfulness-based intervention to control weight after bariatric surgery: Preliminary results from a randomized controlled pilot trial. Complementary Therapies in Medicine, 28, 13-21.

Comunidad de Madrid (2013). Estrategia de atención a pacientes con enfermedades crónicas en la Comunidad de Madrid Madrid: Consejería de Sanidad. Retrieved from http://www.madrid.org/bvirtual/ BVCM017570.pdf

Daubenmier, J., Lin, J., Blackburn, E., Hecht, F. M., Kristeller, J., Maninger, N., Epel, E. (2012). Changes in stress, eating, and metabolic factors are related to changes in telomerase activity in a randomized mindfulness intervention pilot study. Psychoneuroendocrinology, 37(7), 917-928.

Davis, D. M. \& Hayes, J. A. (2011). What are the benefits of mindfulness? A practice review of psychotherapy-related research. Psychotherapy, 48(2), 198-208.

Gainey, A., Himathongkam, T., Tanaka, H. \& Suksom, D. (2016). Effects of Buddhist walking meditation on glycemic control and vascular function in patients with type 2 diabetes. Complementary Therapies in Medicine, 26, 92-97.

Gregg, J. A., Callaghan, G. A., Hayes, S. C. \& Glenn-Lawson, J. L. (2007). Improving diabetes self-management through acceptance, mindfulness, and values: A randomized controlled trial. Journal of Consulting and Clinical Psychology, 75(2), 336-343.

Hartmann, M., Kopf, S., Kircher, C., Faude-Lang, V., Djuric, Z., Augstein, F., Nawroth, P.P. (2012). Sustained Effects of a Mindfulness-Based Stress-Reduction Intervention in Type 2 Diabetic Patients: Design and first results of a randomized controlled trial (the Heidelberger Diabetes and Stress-Study). Diabetes Care, 35(5), 945-947.

International Diabetes Federation. (2015). Atlas de la Diabetes de la FID. Retrieved from https://www.fundaciondiabetes.org/upload/ publicaciones_ficheros/95/IDF_Atlas_2015_SP_WEB_oct2016.pdf

International Diabetes Federation. (2017). Atlas de la Diabetes de la FID. Retrieved from https://www.idf.org/e-library/epidemiology-research/ diabetes-atlas/134-idf-diabetes-atlas-8th-edition.html

Ismail, K., Winkley, K. \& Rabe-Hesketh, S. (2004). Systematic review and meta-analysis of randomised controlled trials of psychological interventions to improve glycaemic control in patients with type 2 diabetes. Lancet, 363(9421), 1589-1597.

Koloverou, E., Tentolouris, N., Bakoula, C., Darviri, C. \& Chrousos, G. (2014). Implementation of a stress management program in outpatients with type 2 diabetes mellitus: a randomized controlled trial. Hormones, 13(4), 509-518.

Liberati, A., Altman, D. G., Tetzlaff, J., Mulrow, C., Gotzsche, P. C., Ioannidis, J.P., Moher, D. (2009). The PRISMA statement for reporting systematic reviews and meta-analyses of studies that evaluate health care interventions: explanation and elaboration. PLoS Medicine, 6(7), e1000100.

Murray, E., Sweeting, M., Dack, C., Pal, K., Modrow, K., Hudda, M., 
Patterson, D. (2017). Web-based self-management support for people with type 2 diabetes (HeLP-Diabetes): randomised controlled trial in English primary care. British Medical Journal Open, 7(9), e016009.

Organización Panamericana de la Salud (2013). Cuidados innovadores para las condiciones crónicas: Organización y prestación de atención de alta calidad a las enfermedades crónicas no transmisibles en las Américas. Retrieved from https://www.paho.org/hq/dmdocuments/2013/ CuidadosInnovadores-v5.pdf

Pearson, S., Wills, K., Woods, M. \& Warnecke, E. (2018). Effects of Mindfulness on Psychological Distress and HbAlc in People with Diabetes. Mindfulness, 9(5), 1615-1626.

Piatt, G. A., Orchard, T. J., Emerson, S., Simmons, D., Songer, T. J., Brooks, M. M., Zgibor, J.C. (2006). Translating the chronic care model into the community: results from a randomized controlled trial of a multifaceted diabetes care intervention. Diabetes Care, 29(4), 811-817.

Putiri, A., Lovejoy, J., Gillham, S., Sasagawa, M., Bradley, R. \& Sun, G.-C. (2012). Psychological Effects of Yi Ren Medical Qigong and Progressive Resistance Training in Adults With Type 2 Diabetes Mellitus: A Randomized Controlled Pilot Study. Alternative Therapies in Health and Medicine, 18(1), 30-34.

Saslow, L.R., Mason, A.E., Kim, S., Goldman, V., Ploutz-Snyder, R., Bayandorian, H., Moskowitz, J. T. (2017). An Online Intervention Comparing a Very Low-Carbohydrate Ketogenic Diet and Lifestyle Recommendations Versus a Plate Method Diet in Overweight Individuals With Type 2 Diabetes: A Randomized Controlled Trial. Journal of Medical Internet Research, 19(2), e36.

Shayeghian, Z., Hassanabadi, H., Aguilar-Vafaie, M.E., Amiri, P. \& Besharat, M.A. (2016). A Randomized Controlled Trial of Acceptance and Commitment Therapy for Type 2 Diabetes Management: The Moderating Role of Coping Styles. PloS One, 11(12), e0166599.

Singh, D. \& Ham, C. (2008). Improving care for people with long term conditions: a review of UK and international frameworks. NHS Institute for Innovation and Improvement. Retrieved from http:// www.improvingchroniccare.org/downloads/review_of_international_ frameworks__chris_hamm.pdf

Smith, J.E., Richardson, J., Hoffman, C. \& Pilkington, K. (2005). Mindfulness-Based Stress Reduction as supportive therapy in cancer care: systematic review. Journal of Advanced Nursing, 52(3), 315-327.

Vandenberg, B.E., Advocat, J., Hassed, C., Hester, J., Enticott, J. \& Russell, G. (2018). Mindfulness-based lifestyle programs for the self-management of Parkinson's disease in Australia. Health Promotion International, $1,9$.

Wagner, E. H. (1998). Chronic disease management: what will it take to improve care for chronic illness? Effective Clinical Practice: ECP, 1(1), 2-4.

Wagner, E.H., Austin, B. T., Davis, C., Hindmarsh, M., Schaefer, J. \& Bonomi, A. (2001). Improving chronic illness care: translating evidence into action. Health Affairs, 20(6), 64-78.

Wagner, E. H., Austin, B. T. \& Von Korff, M. (1996). Organizing care for patients with chronic illness. The Milbank Quarterly, 74(4), 511-544.

Wang, J., Coleman, D. C., Kanter, J., Ummer, B. \& Siminerio, L. (2018).

Connecting Smartphone and Wearable Fitness Tracker Data with a
Nationally Used Electronic Health Record System for Diabetes Education to Facilitate Behavioral Goal Monitoring in Diabetes Care: Protocol for a Pragmatic Multi-Site Randomized Trial. JMIR Research Protocols, 7(4), e10009. 